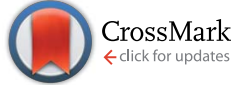

Cite this: Soft Matter, 2014, 10, 9433

Received 27th June 2014

Accepted 23rd September 2014

DOI: $10.1039 / c 4 s m 01409 j$

www.rsc.org/softmatter

\title{
Swim stress, motion, and deformation of active matter: effect of an external field
}

\begin{abstract}
Sho C. Takatori* and John F. Brady
We analyze the stress, dispersion, and average swimming speed of self-propelled particles subjected to an external field that affects their orientation and speed. The swimming trajectory is governed by a competition between the orienting influence (i.e., taxis) associated with the external (e.g., magnetic, gravitational, thermal, nutrient concentration) field versus the effects that randomize the particle orientations (e.g., rotary Brownian motion and/or an intrinsic tumbling mechanism like the flagella of bacteria). The swimmers' motion is characterized by a mean drift velocity and an effective translational diffusivity that becomes anisotropic in the presence of the orienting field. Since the diffusivity yields information about the micromechanical stress, the anisotropy generated by the external field creates a normal stress difference in the recently developed "swim stress" tensor [Takatori, Yan, and Brady, Phys. Rev. Lett., 2014]. This property can be exploited in the design of soft, compressible materials in which their size, shape, and motion can be manipulated and tuned by loading the material with active swimmers. Since the swimmers exert different normal stresses in different directions, the material can compress/expand, elongate, and translate depending on the external field strength. Such an active system can be used as nano/micromechanical devices and motors. Analytical solutions are corroborated by Brownian dynamics simulations.
\end{abstract}

\section{Introduction}

Understanding the complex dynamic behaviors of a suspension of self-propelled colloidal particles, or "active matter," has been an important but challenging problem owing to its constituents' ability to generate their own internal stress and drive the system far from equilibrium. This allows intriguing phenomena to arise that otherwise may not take place in a classical equilibrium system, like athermal self-assembly and pattern formation. ${ }^{1}$ Recently a new principle was introduced to study such fascinating phenomena-that is, through their selfmotion all active matter systems generate an intrinsic "swim stress" that impacts their large-scale collective behavior. ${ }^{2}$ The origin of the swim stress (or pressure) is based upon the notion that all self-propelled bodies must be confined by boundaries to prevent them from swimming away in space. The "swim pressure" is the unique pressure exerted by the swimmers as they bump into the surrounding walls that confine them. The same principle applies to molecular gases that collide into the container walls to exert a pressure or to the osmotic pressure exerted by solute molecules.

In this work we build upon this new perspective to analyze an active matter system subjected to an external field that affects its constituents' swimming orientation and speed. External

Division of Chemistry and Chemical Engineering, California Institute of Technology, Pasadena, CA 91125, USA. E-mail: Takatori@caltech.edu fields like chemical and thermal gradients and/or the Earth's magnetic and gravitational fields can cause microorganisms to modify their swimming behavior to facilitate movement to a favorable region. For example, E. coli have been known to undergo chemotaxis by preferentially swimming towards (or away from) chemical gradients of nutrients (or toxins). ${ }^{3}$ Other examples of taxis swimmers include phototactic, ${ }^{4}$ magnetotactic, ${ }^{5}$ and gravitactic ${ }^{6}$ bacteria.

External orienting fields cause the effective translational diffusivity to become anisotropic, which directly implies the existence of normal stress differences. The micromechanical stress in a dilute suspension is given by the first moment of the force, $\boldsymbol{\sigma}=-n\langle\boldsymbol{x} \boldsymbol{F}\rangle$, where $n$ is the number density of particles and the angle brackets denote an ensemble average over all particles and time. The particle position at time $t$ is $\boldsymbol{x}(t)=\int \boldsymbol{U}\left(t^{\prime}\right) \mathrm{d} t^{\prime}$, and from the overdamped equation of motion, $0=-\zeta \boldsymbol{U}(t)+\boldsymbol{F}(t)$, we obtain $\boldsymbol{\sigma}=-n\langle\boldsymbol{x} \boldsymbol{F}\rangle=-n \zeta \int\left\langle\boldsymbol{U}\left(t^{\prime}\right) \boldsymbol{U}(t)\right\rangle \mathrm{d} t^{\prime}=-n \zeta\langle\boldsymbol{D}\rangle$, where $\zeta$ is the hydrodynamic drag factor and the time integral of the velocity autocorrelation is the diffusivity of the particle, $\boldsymbol{D}$. A particle undergoing any type of random motion therefore exerts a stress and a pressure, $\Pi=-\operatorname{tr} \sigma / 3=n \zeta D$. This general result applies for Brownian particles where $D_{0}=k_{\mathrm{B}} T / \zeta$, leading to the familiar ideal-gas Brownian osmotic pressure $\Pi^{\mathrm{B}}=n k_{\mathrm{B}} T$. Using the swim diffusivity of active particles in the absence of an external field, $\left\langle\boldsymbol{D}^{\text {swim }}\right\rangle=U_{0}{ }^{2} \tau_{\mathrm{R}} \boldsymbol{I} / 6$ where $U_{0}$ is the swim speed of the active particle and $\tau_{\mathrm{R}}$ is the reorientation time due to rotary Brownian motion and/or an intrinsic reorientation 


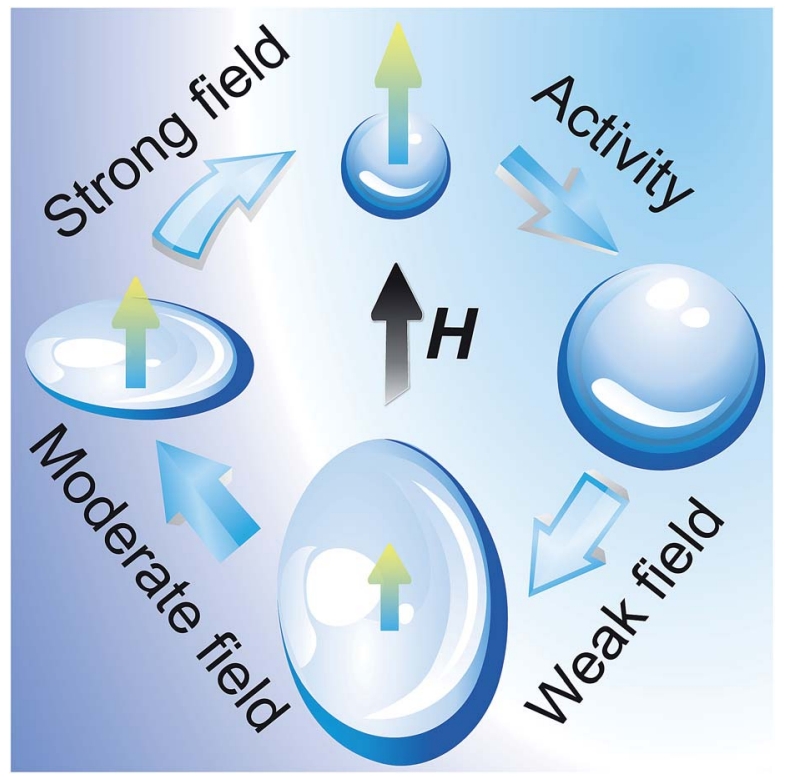

Fig. 1 Schematic of the shape, size, and motion of a soft, compressible gel loaded with light-activated synthetic colloidal particles. When both the light and external field $(H)$ are turned on, the gel translates in the direction of the field (shown by arrows on the gel). The external field strength can be tuned to change the shape, size, and velocity of the gel.

mechanism, we obtain the "ideal-gas" swim stress: $\boldsymbol{\sigma}^{\text {swim }}=-n\left\langle\boldsymbol{x} \boldsymbol{F}^{\text {swim }}\right\rangle=-n \zeta\left\langle\boldsymbol{D}^{\text {swim }}\right\rangle=-n \zeta U_{0}{ }^{2} \tau_{\mathrm{R}} \boldsymbol{I} / 6$, where $F^{\text {swim }} \equiv \zeta U_{0}$ is the self-propulsive force of the swimmer. ${ }^{2}$ Although it is clear that an external field may cause the effective diffusivity and hence the swim stress to become anisotropic, how is this normal swim-stress difference generated and what are its implications on the design of novel active soft-matter materials?

To appreciate the importance of normal swim stresses, we discuss an important application of this work in the design of nano/micromechanical devices and motors. Suppose we load a soft, compressible material (e.g., gel polymer network) with light-activated synthetic colloidal particles. In the absence of light, the colloidal particles simply fluctuate due to Brownian motion, and the gel assumes some equilibrium shape as shown on the top of Fig. 1. The equilibrium volume of the gel is determined by the balance of the force that drives the polymer to expand and mix with the solvent versus the elastic force that resists the expansion. ${ }^{7}$ When the light is turned on, the colloidal particles become active and exert an "ideal-gas" swim pressure, $\Pi^{\text {swim }}=-\operatorname{tr} \boldsymbol{\sigma}^{\text {swim }} / 3=n \zeta U_{0}{ }^{2} \tau_{\mathrm{R}} / 6$, causing the gel to expand isotropically as shown in the sketch on the right. The relative magnitudes of the swim pressure versus the shear modulus of the gel, $G$, determine whether the gel expands appreciably in the presence of the swimmers. In principle the shear modulus of polymer networks can be adjusted to nearly zero. A dilute network of hydrated mucus, which behaves as a non-Newtonian gel, has shear moduli of order $\sim \mathscr{O}(0.1-10) \mathrm{Pa}^{\mathbf{8 , 9}}$ but here we estimate $G \approx n_{\mathrm{c}} k_{\mathrm{B}} T$ where $n_{\mathrm{c}}$ is the number density of subchains in the network (related to the cross-link density). ${ }^{7}$ The energy scale associated with $1 \mu \mathrm{m}$ swimmers traveling in water with speed $U_{0} \sim 10 \mu \mathrm{m} \mathrm{s}^{-1}$ and reorienting in time $\tau_{\mathrm{R}} \sim 10 \mathrm{~s}$ is $\zeta U_{0}{ }^{2} \tau_{\mathrm{R}} / 6 \approx 4 \mathrm{pN} \mu \mathrm{m}$. The thermal energy at room temperature is $k_{\mathrm{B}} T \approx 4 \times 10^{-3} \mathrm{pN} \mu \mathrm{m}$, meaning that the swimmers' intrinsic self-propulsion is equivalent to approximately $1000 k_{\mathrm{B}} T$. In practice the intrinsic activity of active synthetic colloidal particles can be even larger. The swim pressure makes an appreciable contribution to the overall size of the gel if $G / \Pi^{\text {swim }}=n_{\mathrm{c}} k_{\mathrm{B}} T /\left(n 1000 k_{\mathrm{B}} T\right) \lesssim \mathscr{O}(1)$, or when the ratio of the polymer sub-chain density to the active-swimmer density is $n_{\mathrm{c}} / n \lesssim 1000$. The swim pressure exerted at $10 \%$ volume fraction of active particles is $\Pi^{\text {swim }}=n \zeta U_{0}{ }^{2} \tau_{\mathrm{R}} / 6 \approx \mathscr{O}(1)$ Pa. For gels with a very small shear modulus, the swim pressure can cause the gel to deform its shape. As the gel expands due to the swim pressure, the concentration of swimmers decreases. The new volume of the gel is determined by the balance of the gel's expansion forces, the osmotic pressure of the polymer chains, and the swim pressure exerted by the swimmers. Even if the gel does not deform, it can still be translated and be steered using the active swimmers.

As we shall see in Section 6, when we apply a weak external field to the system (gel plus swimmers), the gel reacts in three ways as shown on the bottom of Fig. 1: it expands even more due to an increase in the swim pressure; it elongates in the field direction due to a positive normal stress difference (i.e., the swimmers exert different magnitudes of normal stresses in different directions of the bounding gel network); the entire gel translates in the field direction due to the net motion of the active swimmers colliding into the gel network. Upon further increase in the external field strength, the swim pressure decreases and the normal stress difference becomes negative, which causes the gel to shrink in size, translate faster towards the field direction, and assume the shape of a thin disk as shown on the left of Fig. 1. When the external field strength is made very high, the normal swim-stress difference and swim pressure vanish, causing the gel to return to its equilibrium shape and size but translate in the field direction.

We can make a simple estimate of the gel speed. If an active particle is tethered to a passive particle then it must drag along the passive particle as it swims. The propulsive force available to the swimmer, $\boldsymbol{F}^{\text {swim }} \equiv \boldsymbol{\zeta} \boldsymbol{U}_{0}$, must now balance the combined drag of the swimmer $(-\zeta \boldsymbol{U})$ and its "cargo," which is characterized by a Stokes drag coefficient $\zeta_{\mathrm{C}}$. Thus, the velocity of the combined object is $\boldsymbol{U}=\boldsymbol{\zeta} \boldsymbol{U}_{0} /\left(\zeta_{\mathrm{C}}+\zeta\right)$. If $N$ swimmers are attached the velocity would now go as $\boldsymbol{U}=N \zeta \boldsymbol{U}_{0} /\left(\zeta_{\mathrm{C}}+N \zeta\right)$. The same principle and estimate apply to swimmers confined to a gel. The total propulsive force available is $\boldsymbol{F} \sim n V_{\text {gel }} \zeta\langle\boldsymbol{u}\rangle$, where $V_{\text {gel }}$ is the volume of the gel and $\langle\boldsymbol{u}\rangle$ is the mean swimmer velocity in the presence of the external field (calculated in Sections 4 and 6). This force must balance the gel and swimmers' drag $\boldsymbol{F}^{\mathrm{drag}}=-\left(\zeta_{\text {gel }}+n V_{\text {gel }} \zeta\right) \boldsymbol{U}_{\text {gel }}$ to give $\boldsymbol{U}_{\text {gel }} \sim n V_{\text {gel }}\langle\boldsymbol{u}\rangle \zeta / \zeta_{\text {gel }}\left(1 /\left(1+n V_{\text {gel }} \zeta / \zeta_{\text {gel }}\right)\right)$. The porosity and geometry of the gel would influence $\zeta_{\text {gel }}$, but the drag is proportional to $\boldsymbol{U}_{\mathrm{gel}}$ as in any Stokes-flow problem.

When the external field is turned off, the gel stops translating and an entire cycle is completed as depicted in Fig. 1. Here we have assumed that the active particles are confined to the gel and that the fluid (solvent) is able to flow through the gel as needed. Instead of a gel we can also have a membrane, 


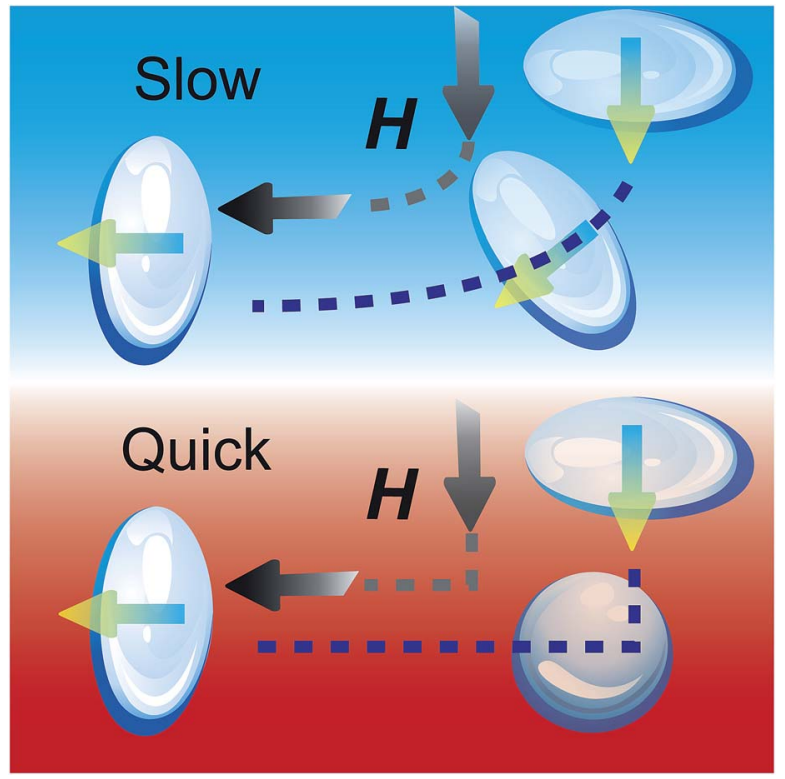

Fig. 2 Schematic of the motion of a soft, compressible gel loaded with active particles when the external field is rotated by 90 degrees. The shape and trajectory of the gel depends on the relative rate of rotation of the field and the strength of the field.

vesicle, fluid sack, or droplet. To ensure that the system is in osmotic balance with the solvent inside the vesicle, the surrounding membrane must be permeable to the solvent. The resistance to motion of the vesicle would now be set by the permeability of the membrane and the propulsive force determined by the number of swimmers contacting the (interior) upstream surface of the vesicle. If we had a vesicle or fluid droplet that is impermeable to the solvent, then the droplet may still deform and may also translate depending on its shape and mechanical properties of its surface or bounding membrane. A rigid object filled with fluid and swimmers would not deform nor translate; the active motion of the swimmers would set up a recirculating flow within the rigid object.

To continue in the design of nano/micromechanical devices and motors, suppose we rotate the external field by 90 degrees. For a moderate external field strength (sketched on the left of Fig. 1), the gel reacts differently depending on the relative magnitude of the characteristic angular velocity induced by the external field, $\Omega_{\mathrm{c}}$, and the rate at which we rotate the field, $\Omega_{\text {ext }}$. When we rotate the field slowly, $\Omega_{\mathrm{ext}} / \Omega_{\mathrm{c}} \ll 1$, the gel maintains its current shape and slowly changes its orientation with the swimmers, tracing an arc and continuing a path along the new field direction, as shown on the top of Fig. 2 . When we rotate the field quickly, $\Omega_{\text {ext }} / \Omega_{\mathrm{c}} \gg 1$, the swimmers respond quickly and begin to swim in the new field direction. In this limit the gel temporarily stops translating because the swimmers do not take any swim steps between their reorientations. After the swimmers change their orientations toward the new field direction, the gel again assumes a disk shape and translates with the swimmers. As illustrated in Fig. 1 and 2, by tuning the properties of the gel (or vesicle or drop), the activity of the swimmers, and the strength of the external orienting field, a wide range of controllable motion is possible. It is important to note that if one can measure the effective translational diffusivity of active particles in an orienting field, then the stress is known from the relationship $\boldsymbol{\sigma}=-n \zeta\langle\boldsymbol{D}\rangle$. We can thus make predictions of the shape and size of the gel based upon a simple diffusivity measurement of the swimmers.

The motion of a single particle due to an intrinsic swim force and an external force are the same. At higher concentrations or when considering the swimmer's interactions with other bodies or boundaries a distinction must be made-the intrinsic swim mechanism does not generate a long-range $1 / r$ Stokes velocity field as does an external force. In our analysis we neglect hydrodynamic interactions among the particles, which would contribute additional terms to the active-particle stress and affect the reorientation time of the particles. It is important to note that the swim stress presented here is distinct and different from the "hydrodynamic stresslet," which is also a single-particle property but scales as $\sim n \zeta U_{0} a$ where $a$ is the particle size. ${ }^{10,11}$ No study to date has studied the effect of an external field on the swim stress of active matter. The ratio of the magnitude of the hydrodynamic stresslet over the swim pressure is the reorientational Péclet number, $\mathrm{Pe}_{\mathrm{R}}=U_{0} a /\left\langle D^{\mathrm{swim}}\right\rangle \sim a /\left(U_{0} \tau_{\mathrm{R}}\right)$, which compares the swimmer size $a$ to its run length $U_{0} \tau_{\mathrm{R}}{ }^{2}$ The hydrodynamic contribution to the deformation of soft materials becomes negligible at low $\mathrm{Pe}_{\mathrm{R}}$, the regime in which many synthetic active particles operate. ${ }^{12,13}$

In this paper we present a micromechanical model that determines the average translational velocity, diffusivity, and swim stress of a suspension of active particles in any external field. Previous studies of the translational diffusivity of Brownian particles have used a generalized Taylor dispersion method to analyze the behavior when subjected to an external orienting field and/or a homogeneous shear flow. ${ }^{14-18}$ Manela and Frankel ${ }^{17}$ analyzed the effective translational diffusivity of dipolar swimmers subjected to a simple shear flow and an external field, and Bearon and coworkers ${ }^{19,20}$ extended the analysis to different flow conditions. Owing to slow numerical convergence, most studies have focused on weak external fields; in practice, however, active particles may be exposed to strong external fields, be it a chemical or thermal gradient field. As shown in this work, strong external fields are interesting because the convective enhancement to the effective translational diffusivity $\left(\left\langle D^{\text {swim }}\right\rangle=U_{0}^{2} \tau_{\mathrm{R}} / 6\right)$ vanishes entirely. Furthermore, most studies assume a constant swimming speed of the particles, irrespective of the external field strength. In nature or in the laboratory, the local chemical and thermal environments can affect the swimming speeds of active particles. Indeed, bacteria modulate their swimming speeds when exposed to a thermal ${ }^{21}$ or chemoattractant concentration field. ${ }^{22}$ We address this problem by allowing the swimmers to modify their speeds based on their instantaneous orientation. Our analytical model is corroborated by Brownian dynamics (BD) simulations.

The balance between the strength of the orienting field and the effects that randomize the particle orientation is characterized by the Langevin parameter, $\chi_{\mathrm{R}}=\Omega_{\mathrm{c}} \tau_{\mathrm{R}}$, where $\Omega_{\mathrm{c}}$ is the characteristic angular velocity induced by the external field and 
$\tau_{\mathrm{R}}$ is the reorientation time from rotary Brownian motion and/or an intrinsic reorientation mechanism. Simple dimensional reasoning provides predictions of the effect of the external field on the average swimming speed, effective translational diffusivity, and swim stress. The self-propulsive enhancement to a swimmer's effective translational diffusivity scales as $\left\langle D^{\text {swim }}\right\rangle \sim L_{\text {eff }}^{2} / \tau_{\mathrm{R}}$, where $L_{\text {eff }}$ is the effective step size. In the absence of an external field $L_{\text {eff }} \sim U_{0} \tau_{\mathrm{R}}$, giving $\left\langle D^{\text {swim }}\right\rangle \sim U_{0}{ }^{2} \tau_{\mathrm{R}}$. With the external field in the linear response regime, the change in the effective step size, $\Delta L_{\text {eff }} \sim \chi_{\mathrm{R}} U_{0} \tau_{\mathrm{R}}$, so the change in swim stress scales as $\Delta \boldsymbol{\sigma}^{\text {swim }}=-n \zeta\left\langle\Delta \boldsymbol{D}^{\text {swim }}\right\rangle \sim-n \zeta\left(U_{0}{ }^{2} \tau_{\mathrm{R}}\right) \chi_{\mathrm{R}}{ }^{2}$. The average velocity along the external field scales as $\left\langle u_{\|}\right\rangle \sim L_{\text {eff }} / \tau_{\mathrm{R}} \sim U_{0} \chi_{\mathrm{R}}$, linear in the forcing. The average velocity transverse to the external field is zero for all values of $\chi_{\mathrm{R}}:\left\langle u_{\perp}\right\rangle=0$. Thus, $\langle\boldsymbol{D}\rangle \sim D_{0}+{U_{0}}^{2} \tau_{\mathrm{R}} / 6\left(1+\mathscr{O}\left(\chi_{\mathrm{R}}{ }^{2}\right)\right)$ and $\boldsymbol{\sigma}^{\text {swim }} \sim-n \zeta U_{0}{ }^{2} \tau_{\mathrm{R}} / 6\left(1+\mathscr{O}\left(\chi_{\mathrm{R}}{ }^{2}\right)\right)$ and is anisotropic.

For $\chi_{\mathrm{R}} \gg 1$, the external field is so strong that the swimmers spend most of their time oriented along the field. This suggests that the average swimmer velocity is $\left\langle u_{\|}\right\rangle \sim U_{0}\left(1-\chi_{\mathrm{R}}{ }^{-1}\right)$; the instantaneous swimmer velocity is the same as the average velocity, minus a small $\mathscr{O}\left(\chi_{\mathrm{R}}{ }^{-1}\right)$ correction. The effective translational diffusivity depends on the fluctuation of the swimmers' instantaneous speed from the average speed. This gives the effective step size, $L_{\text {eff }} \sim\left(\langle u\rangle-U_{0}\right) \tau_{\mathrm{R}}$. Parallel to the external field we thus have $\sigma_{\|}^{\text {swim }}=-n \zeta\left\langle D_{\|}^{\text {swim }}\right\rangle \sim-n \zeta U_{0}{ }^{2} \tau_{\mathrm{R}} \chi_{\mathrm{R}}{ }^{-3}$. In the transverse direction, the average velocity is zero so a small fluctuation in an individual swimmer's perpendicular motion affects the dispersion more strongly than small fluctuations along the external field. This suggests that the swimmer's perpendicular velocity decays more slowly, as $u_{\perp} \sim \mathscr{O}\left(\chi_{\mathrm{R}}{ }^{-1 / 2}\right)$, giving $\sigma_{\perp}^{\text {swim }}=-n \zeta\left\langle D_{\perp}^{\text {swim }}\right\rangle \sim-n \zeta U_{0}{ }^{2} \tau_{\mathrm{R}} \chi_{\mathrm{R}}{ }^{-2}$. Interestingly, under strong external fields the swim stress and diffusivity tend to zero.

In the next section, we formulate an expression for the average translational flux, from which we deduce the swim stress and the average translational velocity and diffusivity. In Section 3, we derive the evolution equations governing the orientation distribution and fluctuation fields. A similar approach has been used to study two-body collisions in nonlinear microrheology, ${ }^{23}$ which we extend here into orientation space. In part 4 , we consider our first example of swimmers with uniform speeds. We build up our BD simulation framework in Section 5 to verify the analytical theory. To obtain a more complete description, in Section 6 we allow the swimming speeds to vary with orientation and field strength.

\section{Average swimmer motion}

We focus on the motion of a single active Brownian particle that swims in a quiescent fluid with an orientation-dependent velocity $\boldsymbol{u}(\boldsymbol{q})$, where the unit vector $\boldsymbol{q}$ specifies its orientation. The swimming velocity can be a result of intrinsic selfpropulsion from a living microorganism or an activated synthetic catalytic particle. ${ }^{\mathbf{1 2 4}}$ The particle also undergoes random thermal motion with a translational diffusivity $\boldsymbol{D}_{0}$, and reorients due to rotary Brownian motion and/or an intrinsic mechanism (e.g., flagella), characterized by a reorientation time

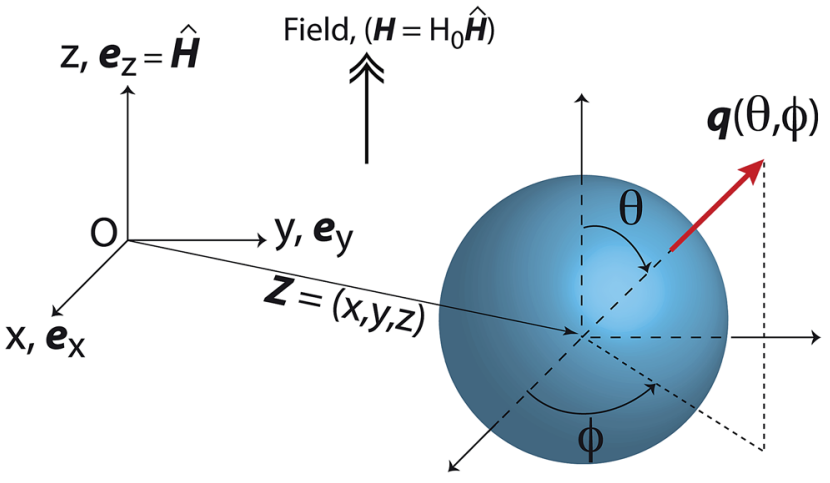

Fig. 3 Definition sketch of an active particle at position $z$ with orientation $q$ in an external field, $H$.

$\tau_{\mathrm{R}}$. For torqued swimmers like gravitactic or magnetotactic bacteria, the external field induces an orientation-dependent torque on the particle, $\boldsymbol{L}^{\text {ext }}(\boldsymbol{q})$. In contrast, force and torquefree swimmers like phototactic bacteria or other microorganisms undergoing chemotaxis or thermotaxis may possess an internal mechanism (e.g., biological sensor) to reorient themselves along the field. Our general analysis remains valid whether the reorientation is induced by an external torque or as a result of an intrinsic particle property. The dynamics of an active particle is contained in $P\left(z, \boldsymbol{q}, t \mid z_{0}, \boldsymbol{q}_{0}, t_{0}\right)$, the conditional probability of finding the particle at position $z$ and orientation $\boldsymbol{q}$ at time $t$, given that it was at $z_{0}$ and $\boldsymbol{q}_{0}$ at time $t_{0}$. This probability density obeys the Smoluchowski equation

$$
\frac{\partial P}{\partial t}+\nabla \cdot \boldsymbol{j}_{\mathrm{T}}+\nabla_{q} \cdot \boldsymbol{j}_{\mathrm{R}}=0
$$

where the translational and rotary fluxes are given by, respectively,

$$
\begin{aligned}
& \boldsymbol{j}_{\mathrm{T}}=\boldsymbol{u}(\boldsymbol{q}) P-\boldsymbol{D}_{0} \cdot \nabla P, \\
& \boldsymbol{j}_{\mathrm{R}}=\boldsymbol{\omega}(\boldsymbol{q}) P-\frac{1}{\tau_{\mathrm{R}}} \nabla_{q} P,
\end{aligned}
$$

where $\omega(q)$ is the orientation-dependent angular velocity of the swimmer, $\boldsymbol{D}_{0}$ is its Brownian translational diffusivity, and $\nabla$ and $\nabla_{q}$ are the physical-space and orientation-space gradient operators, respectively.

We are interested in times $t>\tau_{\mathrm{R}}$ in which all orientations have been sampled. To this end, we follow Zia and Brady ${ }^{23}$ and introduce the Fourier transform with respect to position, denoted by $\wedge$. Averaging eqn (1) and (2) over orientation space, we obtain

$$
\begin{gathered}
\frac{\partial \hat{n}(\boldsymbol{k}, t)}{\partial t}+\mathrm{i} \boldsymbol{k} \cdot\left\langle\hat{j_{\mathrm{T}}}\right\rangle=0, \\
\left\langle\hat{\boldsymbol{j}_{\mathrm{T}}}\right\rangle=\oint \boldsymbol{u} \hat{P} \mathrm{~d} \boldsymbol{q}-\boldsymbol{D}_{0} \cdot i \boldsymbol{k} \hat{n},
\end{gathered}
$$

where $\hat{n}(\boldsymbol{k}, \mathrm{t}) \equiv \oint \hat{P}(\boldsymbol{k}, \boldsymbol{q}, t) \mathrm{d} \boldsymbol{q}$ is the local number density of active particles. We introduce $\hat{P}(\boldsymbol{k}, \boldsymbol{q}, t)=g(\boldsymbol{k}, \boldsymbol{q}, t) \hat{n}(\boldsymbol{k}, t)$, and focus on the orientation distribution through the structure function 
$g(\boldsymbol{k}, \boldsymbol{q}, t)$. For the long-time self-diffusion we consider the short wave vector (long length scale) $\operatorname{limit}^{23}$ and thus expand for small $\boldsymbol{k}: g(\boldsymbol{k}, \boldsymbol{q}, t)=g_{0}(\boldsymbol{q}, t)+\mathrm{i} \boldsymbol{k} \cdot \boldsymbol{d}(\boldsymbol{q}, t)+\mathscr{O}(\boldsymbol{k} \boldsymbol{k})$. The field $g_{0}$ is the orientation distribution function, and $\boldsymbol{d}$ is the probabilityweighted displacement or fluctuation of a particle about its mean velocity (i.e., the strength and direction of the swimmer's displacement due to the external field). Readers familiar with Brenner's ${ }^{25}$ generalized Taylor dispersion theory will notice that $g_{0}$ and $\boldsymbol{d}$ are similar to his $P_{0}^{\infty}$ and $\boldsymbol{B}$ fields, respectively. Introducing this expansion into eqn (5), we obtain the mean particle translational flux:

$$
\left\langle\widehat{\boldsymbol{j}_{\mathrm{T}}}\right\rangle=\hat{n}[\langle\boldsymbol{u}\rangle-\mathrm{i} \boldsymbol{k} \cdot\langle\boldsymbol{D}\rangle]
$$

where the average translational velocity and diffusivity are, respectively,

$$
\begin{gathered}
\langle\boldsymbol{u}\rangle=\oint \boldsymbol{u}(\boldsymbol{q}) g_{0} \mathrm{~d} \boldsymbol{q}, \\
\langle\boldsymbol{D}\rangle-\boldsymbol{D}_{0}=\left\langle\boldsymbol{D}^{\text {swim }}\right\rangle=\oint(\langle\boldsymbol{u}\rangle-\boldsymbol{u}(\boldsymbol{q})) \boldsymbol{d} \mathrm{d} \boldsymbol{q} .
\end{gathered}
$$

In eqn (8) the term $\langle\boldsymbol{u}\rangle$ was inserted to emphasize that it is the velocity fluctuation that generates dispersion.

In the Introduction we derived a direct relationship between the translational diffusivity and the micromechanical stress: $\boldsymbol{\sigma}=-n \zeta\langle\boldsymbol{D}\rangle$. Substituting eqn (8) into this expression gives the stress generated by the active particle, $\boldsymbol{\sigma}=\boldsymbol{\sigma}^{\mathrm{B}}+\boldsymbol{\sigma}^{\text {swim }}$, where we identify the Brownian osmotic stress as $\boldsymbol{\sigma}^{\mathrm{B}}=-n \boldsymbol{\zeta} \boldsymbol{D}_{0}=-n k_{\mathrm{B}} T \boldsymbol{I}$, and the swim stress as the convective enhancement to the diffusivity (right-hand side of eqn (8)):

$$
\boldsymbol{\sigma}^{\text {swim }}=-n \zeta \oint(\langle\boldsymbol{u}\rangle-\boldsymbol{u}(\boldsymbol{q})) \boldsymbol{d} \mathrm{d} \boldsymbol{q} .
$$

Eqn (7) to (9) are the main results we wish to determine. The swim pressure is given by $\Pi^{\mathrm{swim}}=-\mathrm{tr} \boldsymbol{\sigma}^{\mathrm{swim}} / 3$ and is interpreted as the average normal swim stress (i.e., the confinement forces) necessary to prevent an active body from swimming away in space. $^{2}$

\section{Non-equilibrium orientation and fluctuation fields}

We now develop the evolution equations governing the orientation-distribution function $g_{0}$ and the fluctuation field $\boldsymbol{d}$ for use in eqn (7) to (9). From the Smoluchowski eqn (1), $g(\boldsymbol{k}, \boldsymbol{q}, t)$ satisfies

$$
\frac{\partial g}{\partial t}+\nabla_{q} \cdot(\boldsymbol{\omega}(\boldsymbol{q}) g)-\frac{1}{\tau_{\mathrm{R}}} \nabla_{q}^{2} g=g \mathrm{i} \boldsymbol{k} \cdot\left[\langle\boldsymbol{u}\rangle-\boldsymbol{u}(\boldsymbol{q})-\mathrm{i} \boldsymbol{k} \cdot\left(\langle\boldsymbol{D}\rangle-\boldsymbol{D}_{0}\right)\right],
$$

where $g$ is finite on the unit sphere and is normalized: $\oint g(\boldsymbol{k}, \boldsymbol{q}, t) \mathrm{d} \boldsymbol{q}=1$.

To proceed we need a form of $\omega(\boldsymbol{q})$, the rotary velocity that reorients the biased swimmer along the external field or gradient, $\boldsymbol{H}$. For force and torque-free swimmers, like microorganisms undergoing phototaxis, chemotaxis, and/or thermotaxis, we assume that they possess an intrinsic mechanism (e.g., biological sensor) to reorient themselves along $\boldsymbol{H}$. A simple expression for the rotary velocity that models this behavior is $\boldsymbol{\omega}(\boldsymbol{q})=\Omega_{\mathrm{c}} \boldsymbol{q} \times \hat{\boldsymbol{H}}$, where $\Omega_{\mathrm{c}}$ is the magnitude of the angular velocity and $\hat{\boldsymbol{H}}$ is the unit vector along the field. This expression implies that the swimmer attains the maximum rotary velocity when $\boldsymbol{q} \perp \hat{\boldsymbol{H}}$ and zero rotary velocity when $\boldsymbol{q} \| \hat{\boldsymbol{H}}$. Another common class of swimmers, like magnetotactic or gravitactic bacteria, reorient themselves owing to a torque induced by the external field, $\boldsymbol{\omega}(\boldsymbol{q})=\boldsymbol{M}_{\mathrm{R}} \cdot \boldsymbol{L}^{\text {ext }}$, where $\boldsymbol{M}_{\mathrm{R}}$ is the rotary mobility tensor. Following Brenner and Condiff, ${ }^{26}$ one can show that this leads to the same expression as that of the torque-free swimmers. This implies that the detailed reorientation mechanism is unimportant, and both types of swimmers can be modeled with the same expression for the rotary velocity. When analyzing the motion of a single particle, there is no distinction between rotation caused by an external torque and motion arising inherently from the swimmer.

The equations are made dimensionless by scaling $\boldsymbol{u} \sim U_{0}$, $\boldsymbol{\omega}(\boldsymbol{q}) \sim \Omega_{\mathrm{c}}$, and $\boldsymbol{d} \sim U_{0} \tau_{\mathrm{R}}$. Using the small- $k$ expansion and considering a spherical particle with a constant, isotropic Brownian diffusivity, the steady-state orientation distribution function satisfies a convection-diffusion equation:

$$
\nabla_{q}^{2} g_{0}-\chi_{\mathrm{R}} \nabla_{q} \cdot\left[(\boldsymbol{q} \times \hat{\boldsymbol{H}}) g_{0}\right]=0,
$$

with $\oint g_{0} \mathrm{~d} \boldsymbol{q}=1$, and $\chi_{\mathrm{R}} \equiv \Omega_{\mathrm{c}} \tau_{\mathrm{R}}$ is the Langevin parameter. The $\boldsymbol{d}$-field satisfies a similar equation, but is forced by deviations from the mean velocity:

$$
\nabla_{q}^{2} \boldsymbol{d}-\chi_{\mathrm{R}} \nabla_{q} \cdot[(\boldsymbol{q} \times \hat{\boldsymbol{H}}) \boldsymbol{d}]=-g_{0}(\langle\boldsymbol{u}\rangle-\boldsymbol{u}(\boldsymbol{q})) .
$$

\section{Uniform swimming velocity}

In this section, we assume all particles have the nondimensional swim velocity $\boldsymbol{u}(\boldsymbol{q})=\boldsymbol{q}$. We shall see in Section 6 that allowing the speed to change with orientation leads to additional interesting dispersive effects. Eqn (11) and (12) have exact analytical solutions, but we first consider the limiting behaviors at low and high $\chi_{\mathrm{R}}$.

\section{$4.1 \chi_{\mathrm{R}} \ll 1$ limit}

As shown in Appendix A, we apply a regular perturbation to obtain $g_{0}(\boldsymbol{q})=1 /(4 \pi)+\hat{\boldsymbol{H}} \cdot \boldsymbol{P}_{1}(\boldsymbol{q}) \chi_{\mathrm{R}} /(4 \pi)+\hat{\boldsymbol{H}} \hat{\boldsymbol{H}}: \boldsymbol{P}_{2}(\boldsymbol{q}) \chi_{\mathrm{R}}{ }^{2} /(12 \pi)+\mathscr{O}\left(\chi_{\mathrm{R}}{ }^{3}\right)$, where $\boldsymbol{P}_{n}(\boldsymbol{q})$ are the $n^{\text {th }}$-order tensor surface spherical harmonics. ${ }^{27}$ This is identical to Almog and Frankel's ${ }^{15}$ result who considered the sedimentation of axisymmetric noncentrosymmetric particles by gravity. Whether the orienting torque is caused by shape-dependent gravitational settling or from dipole-induced alignment, the orientation distribution is the same.

Substituting this solution into eqn (7), the average translational velocity of the swimmers at low $\chi_{R}$ is 


$$
\langle\boldsymbol{u}\rangle=\frac{1}{3} \chi_{\mathrm{R}} \hat{\boldsymbol{H}}+\mathscr{O}\left(\chi_{\mathrm{R}}^{3}\right) .
$$

The average velocity increases linearly with $\chi_{\mathrm{R}}$, as predicted from simple scaling arguments. As $\chi_{\mathrm{R}} \rightarrow 0$ the orientation distribution becomes uniform, resulting in no net swimming speed.

To obtain a leading-order correction in the swim stress and translational diffusivity, we must proceed to the $\mathscr{O}\left(\chi_{\mathrm{R}}{ }^{2}\right) \boldsymbol{d}$-field problem. Substituting the $\boldsymbol{d}$-field solution (see Appendix A) into eqn (8) and (9), we obtain the swim diffusivity and stress for $\chi_{\mathrm{R}} \ll 1$ :

$$
\begin{aligned}
\boldsymbol{\sigma}^{\text {swim }}= & -n \zeta\left\langle\boldsymbol{D}^{\text {swim }}\right\rangle \\
= & -\frac{n \zeta U_{0}{ }^{2} \tau_{R}}{6}\left[\boldsymbol{I}-\frac{6}{5} \chi_{\mathrm{R}}{ }^{2}\left(\frac{7}{27} \hat{\boldsymbol{H}} \hat{\boldsymbol{H}}+\frac{1}{8}(\boldsymbol{I}-\hat{\boldsymbol{H}} \hat{\boldsymbol{H}})\right)\right] \\
& +\mathscr{O}\left(\chi_{\mathrm{R}}{ }^{4}\right) .
\end{aligned}
$$

We have adopted the transversely isotropic form, where $\hat{\boldsymbol{H}} \hat{\boldsymbol{H}}$ and $\boldsymbol{I}-\hat{\boldsymbol{H}} \hat{\boldsymbol{H}}$ correspond to the parallel and perpendicular components relative to the field direction, respectively. As $\chi_{\mathrm{R}} \rightarrow 0$ we recover the "ideal-gas" swim pressure, $\Pi^{\text {swim }}=n \zeta U_{0}{ }^{2} \tau_{\mathrm{R}} / 6 .{ }^{2}$ The first effect of the external field appears at $\mathscr{O}\left(\chi_{\mathrm{R}}{ }^{2}\right)$, in agreement with our scaling arguments in the Introduction. Notice that the external field causes a decrease in the translational diffusivity, in contrast to the increase seen in the sedimentation problem. ${ }^{\mathbf{1 5}}$ The dispersion decreases here because the particles now swim collectively toward $\hat{\boldsymbol{H}}$, reducing their tendency to take random swim steps.

\section{2 $\chi_{\mathrm{R}} \gg 1$ limit}

A singular perturbation scheme is required for $\chi_{R} \gg 1$ because the problem separates into an outer and inner region. Near $\mu \equiv \hat{\boldsymbol{H}} \cdot \boldsymbol{q} \approx 1$ there is an orientation-space boundary layer and the angular coordinate is rescaled as $\hat{\mu}=(1-\mu) \chi_{\mathrm{R}} \sim \mathscr{O}(1)$. To leading order, $g_{0}$ and $\boldsymbol{d}$ are zero in the outer region because the orientation of the swimmer is confined to a $1 / \chi_{\mathrm{R}^{-}}$thick "cone" around $\mu \approx 1$. As shown in Appendix $\mathrm{B}$, the leading-order boundary-layer solution to eqn (11) is $g_{0}\left(\hat{\mu} ; \chi_{\mathrm{R}}\right)=\chi_{\mathrm{R}} \mathrm{e}^{-\hat{\mu} /(2 \pi)}+\mathscr{O}(1)$. As $\chi_{\mathrm{R}} \rightarrow \infty$, the orientation distribution approaches a delta-function peaked at $\hat{\mu}=0$, confining the swimming trajectory to a narrow "cone" about the field direction. From eqn (7), the average translational velocity is $\langle\boldsymbol{u}\rangle=\left(1-\chi_{\mathrm{R}}{ }^{-1}\right) \hat{\boldsymbol{H}}$. To leading order, all swimmers move along the field direction, $\hat{\boldsymbol{H}}$, at the same speed, $U_{0}$.

The $\boldsymbol{d}$-field problem is resolved into a direction parallel $\left(d_{\|}\right)$ and perpendicular $\left(d_{\perp}\right)$ to the external field. The swim diffusivity and stress for $\chi_{\mathrm{R}} \gg 1$ are

$$
\boldsymbol{\sigma}^{\text {swim }}=-n \zeta\left\langle\boldsymbol{D}^{\text {swim }}\right\rangle=-n \zeta U_{0}{ }^{2} \tau_{\mathrm{R}}\left[\frac{1}{2} \chi_{\mathrm{R}}{ }^{-3} \hat{\boldsymbol{H}} \hat{\boldsymbol{H}}+\chi_{\mathrm{R}}{ }^{-2}(I-\hat{\boldsymbol{H}} \hat{\boldsymbol{H}})\right] .
$$

As $\chi_{\mathrm{R}} \rightarrow \infty$, the swim stress vanishes entirely, including the "ideal-gas" pressure $\Pi^{\text {swim }}=n \zeta U_{0}{ }^{2} \tau_{\mathrm{R}} / 6$ that was present at low $\chi_{\mathrm{R}}$ (see eqn (14)). Since all particles are oriented along a $1 / \chi_{\mathrm{R}}$-thick "cone" about the field, each particle swims at the same velocity $U_{0}$ towards the same direction, resulting in a vanishingly small dispersion. Since it is the random diffusion of a particle that gives rise to a swim pressure, $\Pi^{\text {swim }}=n \zeta \operatorname{tr}\left\langle\boldsymbol{D}^{\text {swim }}\right\rangle / 3$, a small diffusivity results in a small swim pressure. Another way to understand this is to suppose that the bounding walls in a simulation cell were translating with the average particle velocity, $\langle\boldsymbol{u}\rangle$. As $\chi_{\mathrm{R}} \rightarrow \infty$, all particles are swimming with the same speed in the same direction so no confinement pressure is required to contain the particles inside the simulation cell. ${ }^{2}$

\subsection{Exact solution for arbitrary $\chi_{\mathbf{R}}$}

As given in Appendix C, the solution to eqn (11) for arbitrary $\chi_{\mathrm{R}}$ is

$$
g_{0}\left(\mu ; \chi_{\mathrm{R}}\right)=\frac{\chi_{\mathrm{R}}}{4 \pi \sinh \chi_{\mathrm{R}}} \mathrm{e}^{\mu \chi_{\mathrm{R}}}
$$

where $\mu \equiv \hat{\boldsymbol{H}} \cdot \boldsymbol{q}$ as before in the domain $-1 \leq \mu \leq 1$. From eqn (7), the average translational velocity for arbitrary $\chi_{R}$ is

$$
\langle\boldsymbol{u}\rangle=\left(\operatorname{coth} \chi_{\mathrm{R}}-\chi_{\mathrm{R}}{ }^{-1}\right) \hat{\boldsymbol{H}} \equiv \mathscr{L}\left(\chi_{\mathrm{R}}\right) \hat{\boldsymbol{H}},
$$

where $\mathscr{L}\left(\chi_{\mathrm{R}}\right)$ is the Langevin function. As expected, the average perpendicular velocity is zero for all $\chi_{\mathrm{R}}$. We resolve the corresponding displacement field in eqn (12) into the parallel and perpendicular directions. As shown in Appendix C, the parallel direction has an exact solution. In the perpendicular direction, we expand our solution as a series of associated Legendre polynomials. Finally, the effective translational diffusivity and swim stress are obtained from eqn (8) and (9).

\section{Brownian dynamics (BD) simulations}

The motion of active particles in an external field can also be analyzed via BD simulations. The system follows the $N$-particle Langevin equations: $0=-\zeta\left(\boldsymbol{U}-\boldsymbol{U}_{0}\right)+\boldsymbol{F}^{\mathrm{B}}$ and $0=-\boldsymbol{\zeta}_{\mathrm{R}} \boldsymbol{\Omega}+\boldsymbol{L}^{\mathrm{ext}}+\boldsymbol{L}^{\mathrm{R}}$, where $\boldsymbol{U}$ and $\boldsymbol{\Omega}$ are the translational and angular velocities, $\boldsymbol{F}^{\text {swim }} \equiv \zeta \boldsymbol{U}_{0}$ is the self-propulsive force, $\boldsymbol{F}^{\mathrm{B}}$ is the Brownian force, $\zeta_{\mathrm{R}}$ is the hydrodynamic resistance coupling angular velocity to torque, and $\boldsymbol{L}^{\text {ext }}$ and $\boldsymbol{L}^{\mathrm{R}}$ are the torques induced by the external field and rotary Brownian motion and/or an intrinsic reorientation mechanism, respectively. The left-hand sides are zero because inertia is negligible for colloidal dispersions.

The Brownian force and reorientation torque have the white noise statistics $\overline{\boldsymbol{F}^{\mathrm{B}}}=0, \overline{\boldsymbol{F}^{\mathrm{B}}(0) \boldsymbol{F}^{\mathrm{B}}(t)}=2 k_{\mathrm{B}} T \zeta \delta(t) \boldsymbol{I}, \overline{\boldsymbol{L}^{\mathrm{R}}}=0$, and $\overline{\boldsymbol{L}^{\mathrm{R}}(0) \boldsymbol{L}^{\mathrm{R}}(t)}=2 \zeta_{\mathrm{R}}^{2} \delta(t) \boldsymbol{I} / \tau_{\mathrm{R}}$. Particle orientations were updated by relating $\boldsymbol{\Omega}$ to the instantaneous orientation $\boldsymbol{q} \cdot{ }^{28}$ We varied the Langevin parameter $\chi_{\mathrm{R}}$ and analyzed the motion of a single active particle for over 4000 realizations and for at least $100 \tau_{R}$.

The average translational velocity and diffusivity are given by $\langle\boldsymbol{u}\rangle=\mathrm{d}\langle\boldsymbol{x}\rangle / \mathrm{d} t$ and $\langle\boldsymbol{D}\rangle=\lim _{t \rightarrow \infty} \mathrm{d}\left\langle\boldsymbol{x}^{\prime} \boldsymbol{x}^{\prime}\right\rangle /(2 \mathrm{~d} t)$, where $\boldsymbol{x}^{\prime}=\boldsymbol{x}-\langle\boldsymbol{u}\rangle \Delta t$ is the displacement of the swimmer from the mean motion. The swim stress was computed from $\boldsymbol{\sigma}^{\text {swim }}=-n \zeta\left\langle\boldsymbol{x}^{\prime} \boldsymbol{F}^{\text {swim }}\right\rangle$, where $\boldsymbol{F}^{\text {swim' }}=\boldsymbol{F}^{\text {swim }}-\left\langle\boldsymbol{F}^{\text {swim }}\right\rangle$. The average swim force over all 


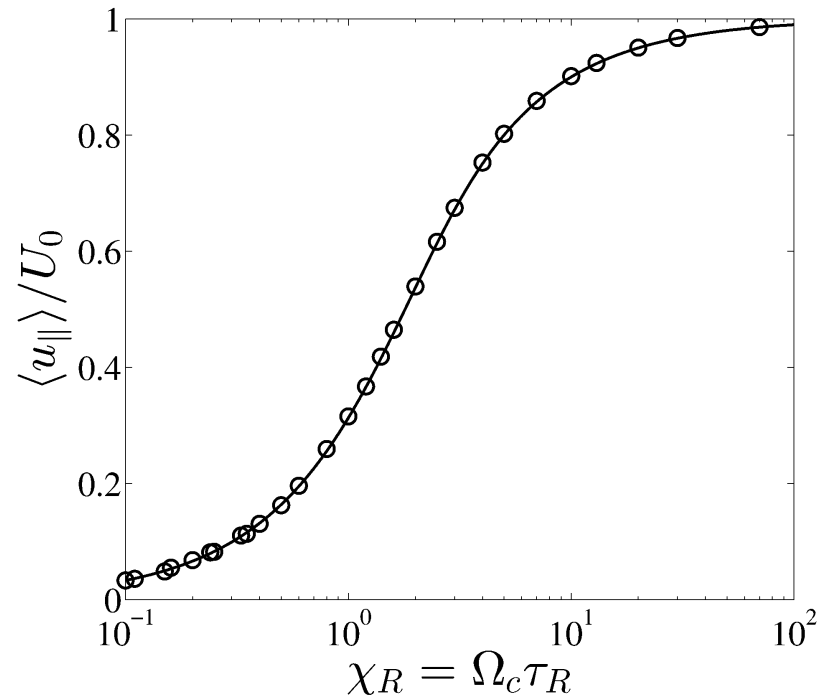

Fig. 4 Average translational velocity along the external field as a function of $\chi_{R}$. The solid curve is the exact analytical solution, and the circles are data from Brownian dynamics (BD) simulations.

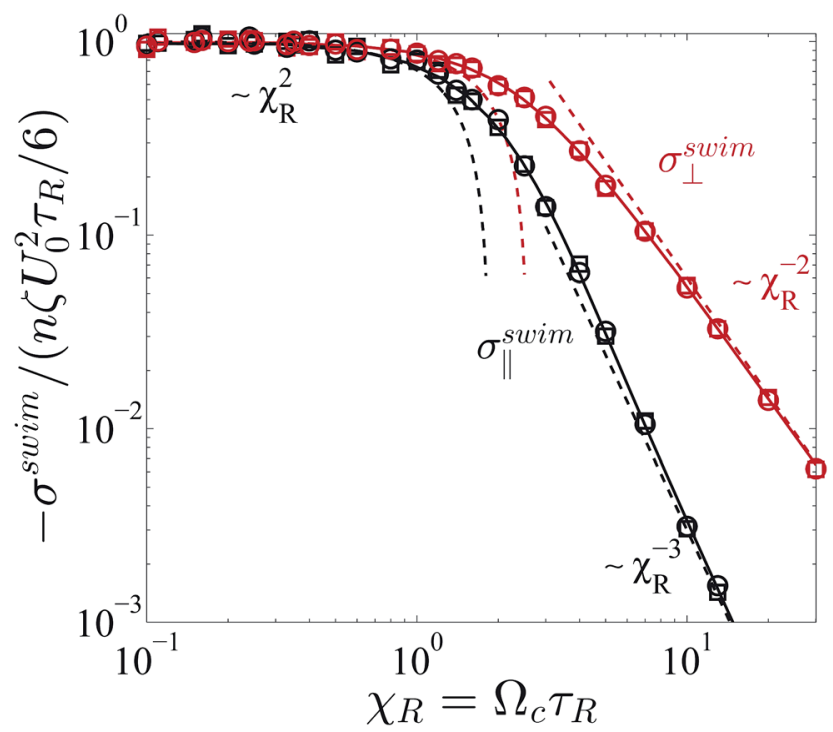

Fig. 5 The swim stress in the parallel (in black) and perpendicular (in red) directions as a function of $\chi_{R}$, computed in the simulations from $\boldsymbol{\sigma}^{\text {swim }}=-n\left\langle\boldsymbol{x}^{\prime} F^{\text {swim }}\right\rangle$ (in circles) and also from first obtaining the effective translational diffusivity and then using $\boldsymbol{\sigma}^{\text {swim }}=-n \zeta\left\langle D^{\text {swim }}\right\rangle$ (in squares). The solid and dashed curves are the exact and asymptotic analytical solutions, respectively.

realizations, $\left\langle\boldsymbol{F}^{\text {swim }}\right\rangle$, must be subtracted to account for the drift velocity of the particles caused by the external field.

\subsection{Results}

Both the asymptotic and exact solutions of the Smoluchowski equation and BD simulation results are presented here together. Fig. 4 shows the nondimensional average swimmer velocity along the external field as a function of $\chi_{\mathrm{R}}$. The average velocity increases linearly following eqn (13) for low $\chi_{\mathrm{R}}$, and

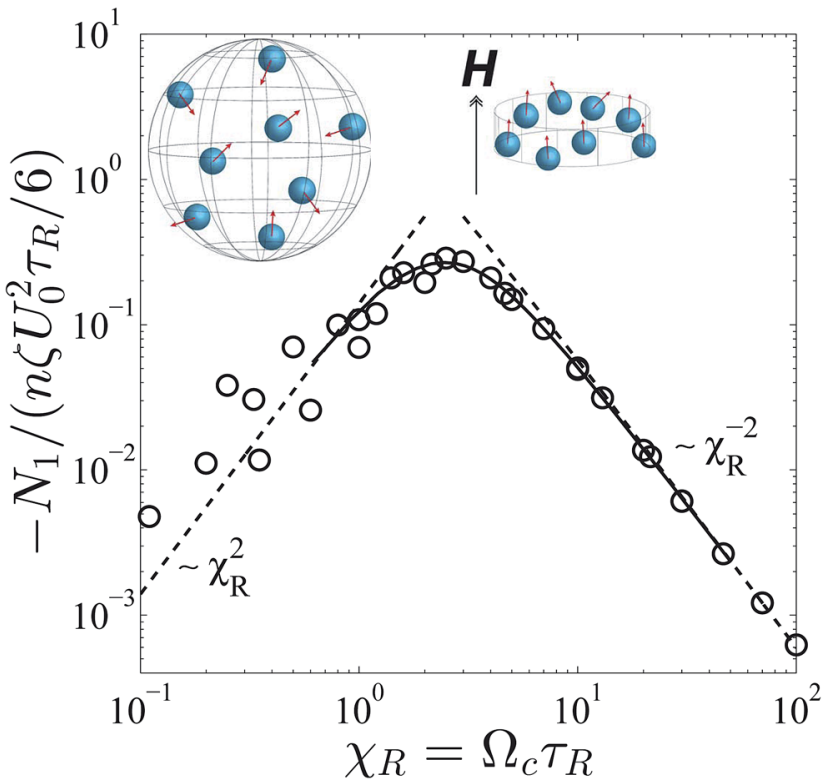

Fig. 6 The first normal swim-stress difference, $N_{1}=\sigma_{\|}^{\text {swim }}-\sigma_{\perp}^{\text {swim }}$, as a function of $\chi_{\mathrm{R}}$. The circles are results from BD simulations, and the solid and dashed curves are the exact and asymptotic analytical solutions, respectively. The illustration shows an instantaneous configuration of the swimmers under a weak (sketch on left) and moderate (on right) external field.

approaches 1 as $\chi_{\mathrm{R}} \rightarrow \infty$. There is no average speed transverse to the external field.

In the BD simulations, the swim stress was computed using two methods. One approach is to use the definition of the swim stress, $\boldsymbol{\sigma}^{\text {swim }}=-n\left\langle\boldsymbol{x}^{\prime} \boldsymbol{F}^{\text {swim' }}\right\rangle$ (shown in circles in Fig. 5). The alternative method is to first calculate the long-time self diffusivity of an active particle and then obtain the swim stress using the relationship $\boldsymbol{\sigma}^{\text {swim }}=-n \zeta\left\langle\boldsymbol{D}^{\text {swim }}\right\rangle$ (shown in squares). The two methods give identical results, verifying that for a single particle the stress is indeed directly related to the diffusivity, $\boldsymbol{\sigma}=-n \zeta\langle\boldsymbol{D}\rangle$. Here we present results for the stress, but the effective translational diffusivity can be obtained by simply dividing the stress by $-n \zeta$.

For $\chi_{\mathrm{R}} \ll 1$, the swim stress reduces to the ideal-gas swim pressure. ${ }^{2}$ The swim stress then decreases as $\sim \mathscr{O}\left(\chi_{\mathrm{R}}{ }^{2}\right)$ following eqn (14). At intermediate values of $\chi_{\mathrm{R}}(\approx 2)$, the curves decline as $\sim \mathscr{O}\left(\chi_{\mathrm{R}}{ }^{-1}\right)$, which means that the dispersion is controlled by convective rotation, i.e., $\boldsymbol{\sigma}^{\text {swim }} \sim-n \zeta{U_{0}}^{2} \tau_{\mathrm{R}} \chi_{\mathrm{R}}{ }^{-1} \sim-n \zeta U_{0}{ }^{2} / \Omega_{\mathrm{c}}$. The diffusivity continues to decay at high $\chi_{\mathrm{R}}$ following eqn (15). An interesting feature at high $\chi_{\mathrm{R}}$ is the faster decay of $\sigma_{\|}^{\text {swim }} \sim \mathscr{O}\left(\chi_{\mathrm{R}}{ }^{-3}\right)$ than $\sigma_{\perp}^{\text {swim }} \sim \mathscr{O}\left(\chi_{\mathrm{R}}{ }^{-2}\right)$. This can be explained by considering the driving force for dispersion, $\Delta \boldsymbol{u}=\langle\boldsymbol{u}\rangle-\boldsymbol{u}(\boldsymbol{q})$. Gradients in $\Delta \boldsymbol{u}$ determine the driving force for dispersion: $\mathrm{d} \Delta u_{\|} / \mathrm{d} \hat{\mu} \sim \chi_{\mathrm{R}}{ }^{-1}$ and $\mathrm{d} \Delta u_{\perp} / \mathrm{d} \hat{\mu} \sim \chi_{\mathrm{R}}{ }^{-1 / 2} \hat{\mu}^{-1 / 2}$. The parallel direction has a small driving force for all $\hat{\mu}$ because an individual particle's instantaneous velocity is the same as the mean, $\left\langle u_{\|}\right\rangle$. A very large fluctuation is required to generate an appreciable contribution to the parallel diffusivity. In contrast, the gradient is maximized at $\hat{\mu}=0$ in the perpendicular direction because the mean transverse velocity is zero. A small fluctuation 


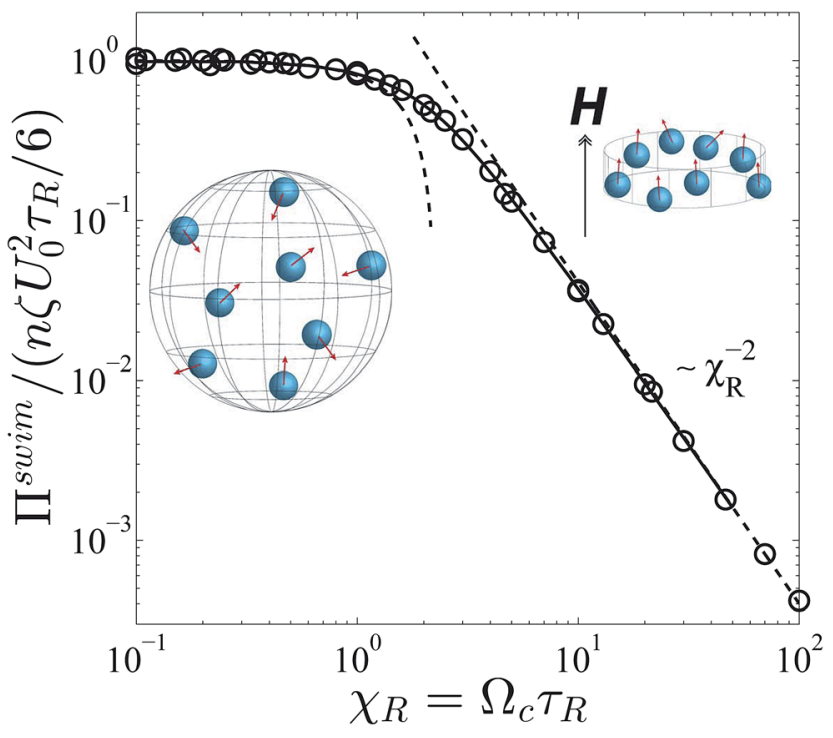

Fig. 7 The swim pressure, $\Pi^{\mathrm{swim}}=-\operatorname{tr} \boldsymbol{\sigma}^{\mathrm{swim}} / 3$, as a function of $\chi_{\mathrm{R}}$. The circles are results from BD simulations, and the solid and dashed curves are the exact and asymptotic analytical solutions, respectively. The illustration shows an instantaneous configuration of the swimmers under a weak (sketch on left) and moderate (on right) external field.

in the perpendicular direction contributes more to the dispersion than in the parallel direction, so $\sigma_{\|}^{\text {swim }}$ decays faster than does $\sigma_{\perp}^{\text {swim }}$.

Fig. 5 shows that the swim stress tensor is anisotropic, which allows us to identify the first normal swim-stress difference: $N_{1}=\sigma_{\|}^{\text {swim }}-\sigma_{\perp}^{\text {swim }}$. Remarkably, this normal swim-stress difference is a single-particle property that arises uniquely from the biased motion of an active particle. As shown in Fig. 6, $N_{1}$ goes to zero for $\chi_{\mathrm{R}} \rightarrow 0$ since the swim stress tensor becomes isotropic. It also goes to zero for $\chi_{\mathrm{R}} \rightarrow \infty$ because the swim stress decays to zero in both the parallel and perpendicular directions (see eqn (15)). It reaches a maximum at intermediate values of $\chi_{\mathrm{R}}$ owing to the rapid decay of the swim stress in the parallel direction $\left(\sigma_{\|}^{\text {swim }} \sim \mathscr{O}\left(\chi_{\mathrm{R}}{ }^{-3}\right)\right)$. Due to axisymmetry the second normal swim-stress difference is zero for all $\chi_{\mathrm{R}}$.

An anisotropic $\boldsymbol{\sigma}^{\text {swim }}$ means that the confining force required to contain the swimmers by the bounding walls would be different in the parallel and perpendicular directions. The swim pressure represents the average of the normal swim stresses (i.e., confinement pressure) exerted on the bounding walls: $\Pi^{\text {swim }}=-\operatorname{tr} \sigma^{\text {swim }} / 3 .^{2}$ As shown in Fig. 7 , the swim pressure approaches the "ideal-gas" value as $\chi_{\mathrm{R}} \rightarrow 0: \Pi^{\mathrm{swim}}=n \zeta U_{0}{ }^{2} \tau_{\mathrm{R}} / 6$. At higher $\chi_{\mathrm{R}}$, the swim pressure decreases since the external field confines the swimming trajectories along the field direction, reducing the confinement pressure on the surrounding walls.

Since normal stress differences indicate how a soft material might elongate or shrink, results from Fig. 6 and 7 can be exploited in the design of various novel active soft materials. Using the results of this section we can now describe how a polymer network (e.g., a gel) loaded with active particles with uniform swim speeds behaves in the presence of an external field. In the absence of the external field, the active particles exert an equal magnitude of normal stress in all directions of the gel, namely $\boldsymbol{\sigma}^{\text {swim }}=-n \zeta U_{0}{ }^{2} \tau_{\mathrm{R}} I / 6$. Upon turning on the external field, the gel shrinks due to the decrease in swim pressure (see Fig. 7), assumes the shape of a thin 3D disk due to the negative normal stress difference (see Fig. 6), and the gel translates due to the average velocity of the swimmers (see Fig. 4). Such a device can be used as a mechanical device/motor where its shape, size, and motion can be carefully tuned by an external field. The gel behavior discussed in the Introduction (Fig. 1) is for non-uniform swim speeds of the particles, which we discuss in Section 6. It is important to note that if one can measure the effective translational diffusivity of active particles in an orienting field, then the stress is known from the relationship $\boldsymbol{\sigma}=-n \zeta\langle\boldsymbol{D}\rangle$. We can thus make predictions of the shape and size of the gel based upon a simple diffusivity measurement of the swimmers.

\section{Nonuniform swimming velocity}

The swimming speeds of bacteria have been shown to change when exposed to chemical $^{22}$ and thermal ${ }^{21}$ gradients. To this end, we now consider the effects of nonuniform swimming speeds on the swim stress and the average translational velocity and diffusivity. Specifically, we allow the swimmers' speed to vary with their orientation relative to the external field, $\boldsymbol{q} \cdot \boldsymbol{H}$. Consider the swimming velocity

$$
\boldsymbol{u}(\boldsymbol{q})=\boldsymbol{q}\left(1+u^{\prime}\left(\alpha H_{0} \hat{\boldsymbol{H}} \cdot \boldsymbol{q}\right)\right)
$$

where $u^{\prime}\left(\alpha H_{0} \hat{\boldsymbol{H}} \cdot \boldsymbol{q}\right)$ is a dimensionless perturbed velocity relative to the uniform speed, $U_{0}$. We introduce $\alpha$ as an intrinsic particle property relating the external field strength, $H_{0}$, to the translational velocity.

The $g_{0}$ solution is identical to eqn (16) since the orientation distribution is independent of $\boldsymbol{u}(\boldsymbol{q})$. However, the $\boldsymbol{d}$-field differs because the driving force $\Delta \boldsymbol{u}=\langle\boldsymbol{u}\rangle-\boldsymbol{u}(\boldsymbol{q})$ is different. Eqn (12) now becomes

$$
\nabla_{q}^{2} \boldsymbol{d}-\chi_{\mathrm{R}} \nabla_{q} \cdot[(\boldsymbol{q} \times \hat{\boldsymbol{H}}) \boldsymbol{d}]=-g_{0}\left[\langle\boldsymbol{u}\rangle-\boldsymbol{q}\left(1+u^{\prime}\left(\alpha H_{0} \hat{\boldsymbol{H}} \cdot \boldsymbol{q}\right)\right)\right],
$$

where

$$
\langle\boldsymbol{u}\rangle=\oint g_{0}\left[\boldsymbol{q}\left(1+u^{\prime}\left(\alpha H_{0} \hat{\boldsymbol{H}} \cdot \boldsymbol{q}\right)\right)\right] \mathrm{d} \boldsymbol{q} .
$$

The swim diffusivity and stress become

$$
\begin{aligned}
\boldsymbol{\sigma}^{\text {swim }} & =-n \zeta\left\langle\boldsymbol{D}^{\text {swim }}\right\rangle \\
& =-n \zeta U_{0}^{2} \tau_{\mathrm{R}} \oint\left[\langle\boldsymbol{u}\rangle-\boldsymbol{q}\left(1+u^{\prime}\left(\alpha H_{0} \hat{\boldsymbol{H}} \cdot \boldsymbol{q}\right)\right)\right] \boldsymbol{d} \mathrm{d} \boldsymbol{q}
\end{aligned}
$$

Eqn (19) and (21) are the only changes required to account for nonuniform swimming speeds. With a choice of $u^{\prime}\left(\alpha H_{0} \hat{\boldsymbol{H}} \cdot \boldsymbol{q}\right)$, the problem statement is complete. Here we 
consider a linear relationship for the velocity perturbation: $u^{\prime}\left(\alpha H_{0} \hat{\boldsymbol{H}} \cdot \boldsymbol{q}\right)=\alpha H_{0} \hat{\boldsymbol{H}} \cdot \boldsymbol{q}$. A swimmer's velocity is now

$$
\boldsymbol{u}(\boldsymbol{q})=\boldsymbol{q}\left[1+\alpha H_{0}(\boldsymbol{q} \cdot \hat{\boldsymbol{H}})\right],
$$

which may be a more complete description than the uniformspeed case considered earlier. When oriented along $\hat{\boldsymbol{H}}$, the swimmer increases its speed, and when oriented antiparallel to $\hat{\boldsymbol{H}}$, it decreases its speed.

Substituting eqn (22) and (16) into eqn (20), the average velocity is

$$
\langle\boldsymbol{u}\rangle=\hat{\boldsymbol{H}}\left[\operatorname{coth} \chi_{\mathrm{R}}-\chi_{\mathrm{R}}{ }^{-1}+\alpha H_{0}\left(1-2 \chi_{\mathrm{R}}{ }^{-1} \operatorname{coth} \chi_{\mathrm{R}}+2 \chi_{\mathrm{R}}{ }^{-2}\right)\right] .
$$

Comparing with eqn (17), we see that the average velocity increases by the last term in parentheses on the right-hand side. At low $\chi_{R}$, the mean velocity of the swimmers is

$$
\langle\boldsymbol{u}\rangle=\hat{\boldsymbol{H}}\left(\frac{1}{3} \alpha H_{0}+\frac{1}{3} \chi_{\mathrm{R}}+\frac{2}{45} \alpha H_{0} \chi_{\mathrm{R}}^{2}+\mathscr{O}\left(\chi_{\mathrm{R}}^{3}\right)\right) .
$$

The first term on the right-hand side represents a mean drift velocity arising from the perturbed velocity. At high $\chi_{\mathrm{R}}$, the swimmers are strongly oriented along the field direction, so the average velocity approaches $U_{0}\left(1+\alpha H_{0}\right)$ following eqn (22).

An analytic solution of eqn (19) for arbitrary $\chi_{\mathrm{R}}$ and $\alpha H_{0}$ is available in Appendix D, but here we analyze the behavior at low and high $\chi_{\mathrm{R}}$. At low $\chi_{\mathrm{R}}$, a regular perturbation scheme gives the swim stress $\boldsymbol{\sigma}^{\text {swim }}=-n \zeta\left[\left\langle D_{\|}^{\text {swim }}\right\rangle \hat{\boldsymbol{H}} \hat{\boldsymbol{H}}+\left\langle D_{\perp}^{\text {swim }}\right\rangle(\boldsymbol{I}-\hat{\boldsymbol{H}} \hat{\boldsymbol{H}})\right]$, where

$$
\begin{aligned}
\left\langle D_{\|}^{\text {swim }}\right\rangle= & U_{0}^{2} \tau_{\mathrm{R}}\left[\left(\frac{1}{6}+\frac{2}{135}\left(\alpha H_{0}\right)^{2}\right)+\frac{2 \chi_{\mathrm{R}}}{27} \alpha H_{0}\right. \\
& \left.-\chi_{\mathrm{R}}^{2}\left(\frac{7}{135}-\frac{\left(\alpha H_{0}\right)^{2}}{189}\right)\right]+\mathscr{O}\left(\chi_{\mathrm{R}}^{3}\right), \\
\left\langle D_{\perp}^{\text {swim }}\right\rangle= & U_{0}^{2} \tau_{\mathrm{R}}\left[\left(\frac{1}{6}+\frac{1}{90}\left(\alpha H_{0}\right)^{2}\right)+\frac{\chi_{\mathrm{R}}}{18} \alpha H_{0}\right. \\
& \left.-\chi_{\mathrm{R}}{ }^{2}\left(\frac{1}{40}-\frac{59\left(\alpha H_{0}\right)^{2}}{22680}\right)\right]+\mathscr{O}\left(\chi_{\mathrm{R}}{ }^{3}\right) .
\end{aligned}
$$

As $\alpha H_{0} \rightarrow 0$, the results reduce to the uniform-speed solution considered earlier. The striking feature is that the dispersion increases at small $\chi_{\mathrm{R}}$, unlike the uniform-velocity case (compare with eqn (14)). Since the swimmers oriented towards the field move faster than those oriented away from the field, we see an enhanced dispersion (and swim stress) at low to intermediate $\chi_{\mathrm{R}}$. As we shall see from the exact solution, the swim stresses in both parallel and perpendicular directions continue to increase and reach a maximum at intermediate $\chi_{R}$.

Another key difference is the anisotropic swim stress at $\chi_{\mathrm{R}}=0$; the parallel diffusion is larger $(2 / 135$ versus $1 / 90$ for $\left.\alpha H_{0}=1\right)$. The average drift velocity from eqn (24) increases the effective translational diffusivity above $U_{0}^{2} \tau_{\mathrm{R}} / 6$ even at $\chi_{\mathrm{R}}=0$.
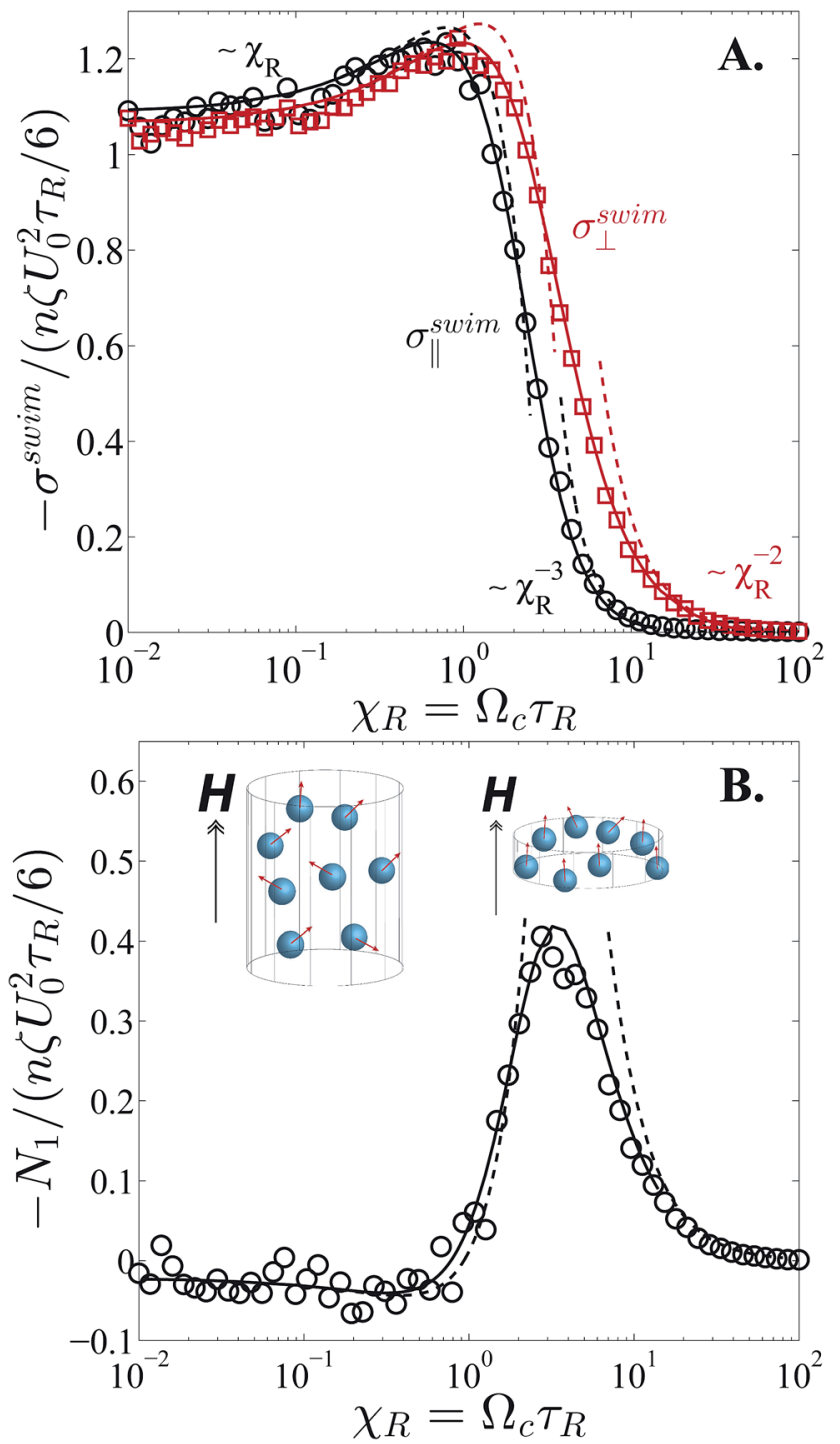

Fig. 8 (A) Swim stress in the parallel (in black) and perpendicular (in red) directions as a function of $\chi_{R}$ for $\alpha H_{0}=1$. The $\alpha H_{0}$ parameter allows the swimming speed to vary with particle orientation. (B) First normal swim-stress difference. The illustration shows an instantaneous configuration of the swimmers under a weak (sketch on left) and moderate (on right) external field. In both (A) and (B), the solid curves are the exact solutions, and the dashed curves are the asymptotic solutions. In (A) BD simulation results are shown in circles and squares for the parallel and perpendicular directions, respectively.

This drift velocity may help explain the observed migration of bacteria along a temperature gradient. ${ }^{21}$

At high $\chi_{\mathrm{R}}$, the behavior is similar to the uniform-velocity case. Since all particles are oriented along the external field, the effect of swimming-speed nonuniformity becomes negligible and the particles swim in the same direction with the same speed. The swim stress at high $\chi_{\mathrm{R}}$ is

$$
\begin{aligned}
\boldsymbol{\sigma}^{\text {swim }}= & -n \zeta U_{0}{ }^{2} \tau_{\mathrm{R}}\left[\frac{1}{2}\left(1+2 \alpha H_{0}\right)^{2} \chi_{\mathrm{R}}{ }^{-3} \hat{\boldsymbol{H}} \hat{\boldsymbol{H}}\right. \\
& \left.+\left(1+\alpha H_{0}\right)^{2} \chi_{\mathrm{R}}{ }^{-2}(\boldsymbol{I}-\hat{\boldsymbol{H}} \hat{\boldsymbol{H}})\right] .
\end{aligned}
$$




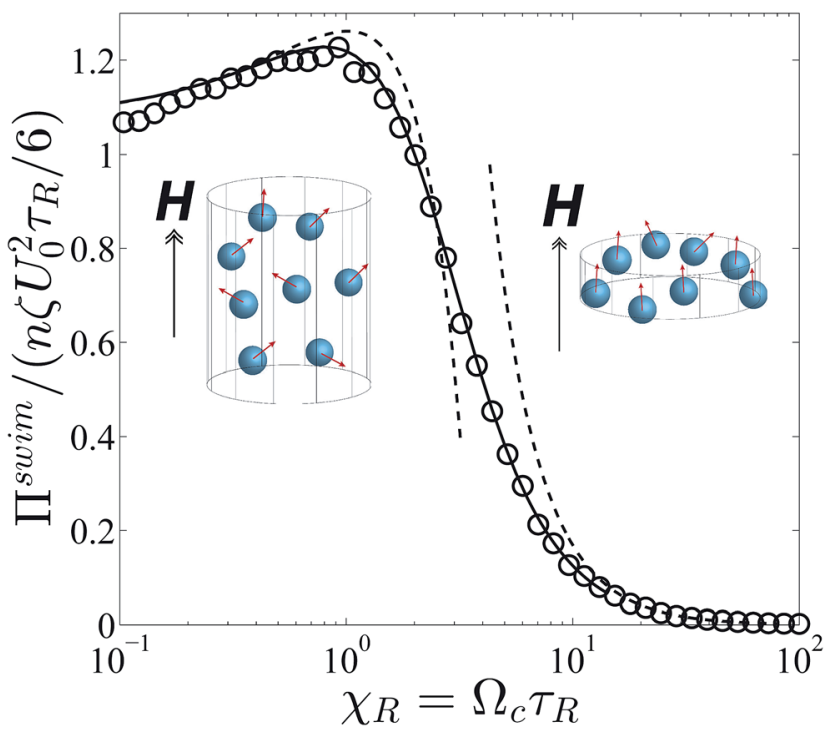

Fig. 9 The swim pressure, $\Pi^{\mathrm{swim}}=-\operatorname{tr} \sigma^{\mathrm{swim}} / 3$, as a function of $\chi_{\mathrm{R}}$ for $\alpha H_{0}=1$. The circles are results from BD simulations, and the solid and dashed curves are the exact and asymptotic analytical solutions, respectively. The illustration shows an instantaneous configuration of the swimmers under a weak (sketch on left) and moderate (on right) external field.

The swim stress as a function of $\chi_{\mathrm{R}}$ for $\alpha H_{0}=1$ is shown in Fig. 8A. The instantaneous swimming speed is twice the uniform speed when the swimmer is oriented along the field $\left(2 U_{0}\right)$ and zero when oriented in the opposite direction. The swim stress increases at low to moderate $\chi_{\mathrm{R}}$ and reaches a maximum at $\chi_{\mathrm{R}}^{\max }=0.60$ and $\chi_{\mathrm{R}}^{\max }=0.95$ in the parallel and perpendicular directions, respectively. We see maxima because the field redistributes the orientations and modifies the swimming speeds. This is different from the uniform-speed case where the field affected only the swimming orientations. As shown in Fig. 8B, the normal swim-stress difference is nonmonotonic and also changes in sign from negative to positive at $\chi_{\mathrm{R}} \approx 0.8$.

We saw in Fig. 7 that an external field that affects the particles' swimming orientation (but not their speed) resulted in a monotonically decreasing swim pressure with $\chi_{\mathrm{R}}$. As shown in Fig. 9, the swim pressure becomes non-monotonic when both the particles' swimming orientation and speed are affected by the external field. This is interesting because an external field can give a non-monotonic pressure profile at the single-particle level (i.e., an infinitely dilute system).

In the Introduction we discussed an important application of loading a soft, compressible gel with active particles. Here we support the description of Fig. 1 with our results. When the colloidal particles are inactive, the gel assumes some equilibrium shape as shown on the top of Fig. 1. Activating the colloidal particles causes the gel to swell due to the "ideal-gas" swim pressure of the active particles, $\Pi^{\text {swim }}=n \zeta U_{0}{ }^{2} \tau_{\mathrm{R}} / 6$. Since the shear modulus of polymer networks can be adjusted over a wide range (in principle to nearly zero) and the intrinsic activity of the swimmers can be made much larger than the thermal energy, $\zeta U_{0}{ }^{2} \tau_{\mathrm{R}} \gg k_{\mathrm{B}} T$, the swim pressure can make an appreciable contribution to the overall size of the gel.

When we then apply a weak external field (i.e., $\chi_{\mathrm{R}}<1$ ), the gel expands even more due to increased swim pressures (see Fig. 9), elongates due to positive normal stress differences (see Fig. 8B), and translates due to the net motion of the active swimmers (see eqn (24)) within the gel. When we increase the external field strength $\left(1<\chi_{\mathrm{R}} \ll \infty\right)$, the swim pressure decreases and the normal stress difference becomes negative (Fig. 8B graphs $-N_{1}$ ), which causes the gel to shrink in size, translate faster towards the field direction, and assume the shape of a thin disk as shown on the left of Fig. 1. When the external field strength becomes very high $\left(\chi_{\mathrm{R}} \rightarrow \infty\right)$, the normal swim-stress difference and swim pressure vanish, causing the gel to return to its equilibrium shape and size but translate in the field direction. When the external field is turned off, the gel stops translating and an entire cycle is completed as depicted in Fig. 1. Each transformation of the gel is corroborated by our calculations and BD simulations.

Allowing the swimming speeds to vary with orientation introduces features similar to the sedimentation problem considered by Brenner ${ }^{14}$ and Almog and Frankel. ${ }^{15}$ In the effective translational diffusivity (eqn (25) and (26)), the terms involving $\left(\alpha H_{0}\right)^{2}$ are identical to those by Almog and Frankel. ${ }^{15}$ When analyzing the motion of a single particle, there is no distinction between a motion caused by an external force (i.e., gravity) and a motion arising from intrinsic particle activity (i.e., swim force). Therefore, the perturbation $u^{\prime}=\alpha H_{0} \hat{\boldsymbol{H}} \cdot \boldsymbol{q}$ in the modified velocity expression is similar to adding a contribution from an external force, $\boldsymbol{M}(\boldsymbol{q}) \cdot \boldsymbol{F}^{\mathrm{ext}}$, where $\boldsymbol{M}(\boldsymbol{q})$ is the orientation-dependent mobility and $\boldsymbol{F}^{\text {ext }}$ is the external force. Of course, one could assume an expression of $u^{\prime}\left(\alpha H_{0} \hat{\boldsymbol{H}} \cdot \boldsymbol{q}\right)$ that is different from the linear relationship (eqn (22)) considered here, and the results would no longer be the same as the sedimentation problem. Therefore, for a single particle the sedimentation problem is a special case of our general formulation.

\section{Conclusions}

We have introduced a new approach to understand and compute the active stress in a system of self-propelled bodies. All active matter systems generate a unique swim pressure through their intrinsic self-motion. Here we used this swim stress perspective to analyze the effect of an external field on the motion and deformation of active matter. We saw that the external field engendered anisotropic stresses, meaning that the swimmers experience a different confining force in the parallel and perpendicular directions. This lead directly to the shrinking/expanding, elongating, and translating of soft, compressible materials that are loaded with active particles. The external field can thus be used to manipulate the shape and size of soft materials such as a gel or perhaps a biological membrane. Another important application may be the analysis of various biophysical systems, such as the interior of a cell. Molecular motors that activate the cytoskeleton must exert a 
swim pressure on the cell owing to their self-motion along a track.

Our analysis remains valid for non-spherical particles with a varying swim velocity $\boldsymbol{U}_{0}$ and/or reorientation time $\tau_{\mathrm{R}}$. Here we focused on a dilute system of swimmers, but inclusion of twobody effects in the Smoluchowski eqn (1) is straightforward. For non-spherical particles the hydrodynamic drag tensor, $\zeta$, varies with the director $\boldsymbol{q}$, and the effective hydrodynamic drag factor $\zeta^{\text {eff }}=\left(2 \zeta_{\perp}+\zeta_{\|}\right) / 3$ becomes the relevant quantity in the stress-diffusivity relationship, where $\zeta_{\perp}$ and $\zeta_{\|}$are the transverse and parallel components of the hydrodynamic drag tensor, respectively. At finite volume fractions, the particle size, $a$, would enter in the form of a nondimensional rotary Péclet number, $\mathrm{Pe}_{\mathrm{R}}=U_{0} a /\left\langle D^{\text {swim }}\right\rangle \sim a /\left(U_{0} \tau_{\mathrm{R}}\right)$, which compares the swimmer size $a$ to its run length $U_{0} \tau_{\mathrm{R}}$. With the inclusion of translational Brownian motion, all three parameters must be varied in the analysis: $\chi_{\mathrm{R}}=\Omega_{\mathrm{c}} \tau_{\mathrm{R}}, \mathrm{Pe}_{\mathrm{R}}=a /\left(U_{0} \tau_{\mathrm{R}}\right)$, and the swim Péclet number $\mathrm{Pe}_{\mathrm{s}}=U_{0} a / D_{0}$.

In our analysis we neglected hydrodynamic interactions among the particles, which would contribute additional terms to the active-particle stress and affect the reorientation time of the particles due to translation-rotation coupling. It is important to note that the swim stress is distinct and different from the "hydrodynamic stresslet", which is also a single-particle property but scales as $\sim n \zeta U_{0} a .^{\mathbf{1 0 , 1 1}}$ As mentioned before, the motion of a single particle due to an intrinsic swim force and an external force are the same. At higher concentrations or when considering the swimmer's interactions with other bodies or boundaries a distinction must be made-the intrinsic swim mechanism does not generate a long-range $1 / r$ Stokes velocity field as does an external force.

Here we focused on a dilute system of active particles, but at higher concentrations active systems have been known to exhibit unique collective behavior.,29 The swim pressure presented here remains valid and appropriate for hydrodynamically interacting active systems, but one needs to carefully examine the individual contributions to the active stress. A single particle hydrodynamic contribution to the stress is of the form $\sim n \zeta a \boldsymbol{U}$, which, while important, is much smaller by a factor of $U_{0} \tau_{\mathrm{R}} / a$ than the swim pressure. A complete study would need to consider the effects of both the swim and hydrodynamic stresses. We believe that the experimental, numerical, and theoretical analyses of active systems may need to be revisited in light of the new swim stress concept.

Experimentally, the precise manipulation of colloids using external fields is critical in many applications, like the targeted transport and delivery of specific chemicals. ${ }^{30}$ Active-matter systems are ideal candidates for understanding dynamic selfassembly and developing synthetic structures. For example, dipolar particles subjected to a magnetic or electric field have been shown to form patterns. ${ }^{30-32}$ Self-assembly and clustering behavior in active matter have been analyzed from the swim stress perspective, ${ }^{2}$ and it would be straightforward to extend these ideas to self-propelled particles that are biased by an external orienting field.

\section{Appendix}

\section{(A) Low- $\chi_{\mathbf{R}}$ limit}

A regular perturbation expansion of eqn (11) and (12) assumes solutions of the form $g_{0}\left(\boldsymbol{q} ; \chi_{\mathrm{R}}\right)=g_{0}^{(0)}(\boldsymbol{q})+g_{0}^{(1)}(\boldsymbol{q}) \chi_{\mathrm{R}}+g_{0}^{(2)}(\boldsymbol{q}) \chi_{\mathrm{R}}{ }^{2}+\mathscr{O}\left(\chi_{\mathrm{R}}{ }^{3}\right)$ and $\boldsymbol{d}\left(\boldsymbol{q} ; \chi_{\mathrm{R}}\right)=\boldsymbol{d}^{(0)}(\boldsymbol{q})+\boldsymbol{d}^{(1)}(\boldsymbol{q}) \chi_{\mathrm{R}}+\boldsymbol{d}^{(2)}(\boldsymbol{q}) \chi_{\mathrm{R}}^{2}+\mathscr{O}\left(\chi_{\mathrm{R}}^{3}\right)$.

Substituting these into eqn (11) of the text, the leading-order orientation distribution function $g_{0}^{(0)}$ satisfies $\nabla_{q}^{2} g_{0}^{(0)}=0$ and $\oint_{0}^{(0)} \mathrm{d} \boldsymbol{q}=1$. The solution is the uniform distribution, $g_{0}^{(0)}=1 / 4 \pi$. The $\mathscr{O}\left(\chi_{\mathrm{R}}\right)$ problem is $-\hat{\boldsymbol{H}} \cdot \boldsymbol{q} /(2 \pi)=\nabla_{q}^{2} \mathrm{~g}_{0}^{(1)}$ with $\oint g_{0}^{(1)} \mathrm{d} \boldsymbol{q}=0$. From Brenner, ${ }^{27}$ vector spherical surface harmonics satisfy

$$
\nabla_{q}^{2} \boldsymbol{P}_{n}(\boldsymbol{q})=-n(n+1) \boldsymbol{P}_{n}(\boldsymbol{q})
$$

We hence substitute the trial solution $g_{0}^{(1)}=\boldsymbol{P}_{1}(\boldsymbol{q}) \cdot \boldsymbol{a}_{1}$ into eqn (A1), and obtain $\boldsymbol{a}_{1}=\hat{\boldsymbol{H}} / 4 \pi$. Thus, the solution is $\mathrm{g}_{0}^{(1)}=\hat{\boldsymbol{H}} \cdot \boldsymbol{P}_{1}(\boldsymbol{q}) /(4 \pi)$. The $\mathscr{O}\left(\chi_{\mathrm{R}}^{2}\right)$ problem is solved similarly: $\nabla_{q}^{2} \mathrm{~g}_{0}^{(2)}=-\hat{\boldsymbol{H}} \hat{\boldsymbol{H}}: \boldsymbol{P}_{2}(\mathbf{q}) /(2 \pi)$ with $\oint g_{0}^{(2)} \mathrm{d} \boldsymbol{q}=0$. The solution is $g_{0}^{(2)}=\hat{\boldsymbol{H}} \hat{\boldsymbol{H}}: \boldsymbol{P}_{2}(\boldsymbol{q}) /(12 \pi)$. Substitution of these three contributions into the perturbation expansion, we arrive at the solution in the text.

A similar procedure for the $\boldsymbol{d}$-field gives

$$
\begin{aligned}
\boldsymbol{d}= & -\frac{1}{8 \pi} \boldsymbol{P}_{1}(\boldsymbol{q})-\frac{5 \chi_{\mathrm{R}}}{72 \pi} \hat{\boldsymbol{H}} \cdot \boldsymbol{P}_{2}(\boldsymbol{q}) \\
& +\frac{\chi_{\mathrm{R}}{ }^{2}}{\pi}\left(\frac{29}{1440} \hat{\boldsymbol{H}} \hat{\boldsymbol{H}} \cdot \boldsymbol{P}_{1}(\boldsymbol{q})-\frac{13}{720} \hat{\boldsymbol{H}} \hat{\boldsymbol{H}}: \boldsymbol{P}_{3}(\boldsymbol{q})-\frac{3}{160} \boldsymbol{P}_{1}(\boldsymbol{q})\right) \\
& +\mathscr{O}\left(\chi_{\mathrm{R}}^{3}\right) .
\end{aligned}
$$

As in the force-induced microrheology problem considered by Zia and Brady, ${ }^{23}$ the $\mathscr{O}(1)$ solution for $\boldsymbol{d}$ is the same as the $\mathscr{O}\left(\chi_{\mathrm{R}}\right)$ problem for $g_{0}$. In the linear-response regime, the problems are identical whether the swimmers are reoriented by the external field $\left(g_{0}\right)$ or by thermal energy $k_{\mathrm{B}} T(\boldsymbol{d})$ and the same holds true when the reorientation is athermal with $\tau_{R}$.

\section{(B) High- $\chi_{\mathbf{R}}$ limit}

The problem is singular in the $\chi_{\mathrm{R}} \gg 1$ limit, so we expand the solution in the inner region as $g_{0}\left(\hat{\mu} ; \chi_{\mathrm{R}}\right)=\chi_{\mathrm{R}} g_{0}^{(0)}(\hat{\mu})+g_{0}^{(1)}(\hat{\mu})+\mathscr{O}\left(\chi_{\mathrm{R}}{ }^{-1}\right)$. Substituting into eqn (11) of the text, the leading-order solution satisfies

$$
\frac{\mathrm{d}}{\mathrm{d} \hat{\mu}}\left[\hat{\mu}\left(g_{0}^{(0)}+\frac{\mathrm{d} g_{0}^{(0)}}{\mathrm{d} \hat{\mu}}\right)\right]=0
$$

with $\int_{0}^{2 \pi} \int_{0}^{\infty} g_{0}^{(0)}(\hat{\mu}) \mathrm{d} \hat{\mu} \mathrm{d} \phi=1$. For the fluctuation field, we separate the solution into scalar components parallel and perpendicular to $\hat{\boldsymbol{H}}$ as $\boldsymbol{d}\left(\mu, \phi ; \chi_{\mathrm{R}}\right)=d_{\|}\left(\mu ; \chi_{\mathrm{R}}\right) \hat{\boldsymbol{H}}+d_{\perp}\left(\mu ; \chi_{\mathrm{R}}\right)\left(\boldsymbol{e}_{x} \cos \phi+\boldsymbol{e}_{y} \sin \phi\right)$, where $\boldsymbol{e}_{x}$ and $\boldsymbol{e}_{y}$ are unit vectors in the $x$ and $y$ directions, respectively (see Fig. 3). We assume subject to posteriori verification that $d_{\|}$and $d_{\perp}$ are only a function of $\mu$. Substituting the scaled $\hat{\mu}$ variable into eqn (12), we obtain

$$
\frac{\mathrm{d}}{\mathrm{d} \hat{\mu}}\left[\hat{\mu}\left(d_{\|}+\frac{\mathrm{d} d_{\|}}{\mathrm{d} \hat{\mu}}\right)\right]=-\frac{1}{4 \pi} \mathrm{e}^{-\hat{\mu}} \chi_{\mathrm{R}}{ }^{-1}(\hat{\mu}-1),
$$




$$
\frac{\mathrm{d}}{\mathrm{d} \hat{\mu}}\left[\hat{\mu}\left(d_{\perp}+\frac{\mathrm{d} d_{\perp}}{\mathrm{d} \hat{\mu}}\right)\right]-\frac{d_{\perp}}{4 \hat{\mu}}=\frac{\sqrt{2}}{4 \pi} \chi_{\mathrm{R}}^{-1 / 2} \mathrm{e}^{-\hat{\mu}} \hat{\mu}^{1 / 2}
$$

The leading nonzero solution is of order $d_{\|} \sim \mathscr{O}\left(\chi_{\mathrm{R}}{ }^{-1}\right)$ and $d_{\perp} \sim \mathscr{O}\left(\chi_{\mathrm{R}}{ }^{-1 / 2}\right)$. In the parallel direction, the solution is $d_{\|}\left(\hat{\mu} ; \quad \chi_{\mathrm{R}}\right)=\chi_{\mathrm{R}}{ }^{-1} \mathrm{e}^{-\hat{\mu}}(\hat{\mu}-1) /(4 \pi)+\mathscr{O}\left(\chi_{\mathrm{R}}{ }^{-2}\right)$, which satisfies both the regularity and normalization conditions. In the perpendicular direction, we obtain $d_{\perp}\left(\hat{\mu} ; \chi_{\mathrm{R}}\right)=-\chi_{\mathrm{R}}{ }^{-1 / 2} \hat{\mu}^{1 / 2} \mathrm{e}^{-\hat{\mu}} /(\sqrt{2} \pi)+\mathscr{O}\left(\chi_{\mathrm{R}}{ }^{-1}\right)$. Using boundary-layer coordinates, the effective translational diffusivity is computed from

$$
\begin{aligned}
\langle\boldsymbol{D}\rangle-\boldsymbol{D}_{0}=\left\langle\boldsymbol{D}^{\mathrm{swim}}\right\rangle= & \pi U_{0}{ }^{2} \tau_{\mathrm{R}} \int_{0}^{\infty}\left[2 \chi_{\mathrm{R}}{ }^{-2}(1-\hat{\mu}) d_{\|} \hat{\boldsymbol{H}} \hat{\boldsymbol{H}}\right. \\
& \left.+\sqrt{2} \chi_{\mathrm{R}}{ }^{-3 / 2} d_{\perp} \hat{\mu}^{1 / 2}(\boldsymbol{I}-\hat{\boldsymbol{H}} \hat{\boldsymbol{H}})\right] \mathrm{d} \hat{\mu} .
\end{aligned}
$$

\section{(C) Exact solution for arbitrary $\chi_{\mathrm{R}}$ : uniform speeds}

We rewrite eqn (11) as

$$
\frac{\mathrm{d}}{d \mu}\left[\left(1-\mu^{2}\right) \frac{\mathrm{d} g_{0}}{\mathrm{~d} \mu}\right]-\chi_{\mathrm{R}} \frac{\mathrm{d}}{\mathrm{d} \mu}\left[\left(1-\mu^{2}\right) g_{0}\right]=0,
$$

where $\mu \equiv \hat{\boldsymbol{H}} \cdot \boldsymbol{q}$. Twice integrating and invoking the normalization and regularity conditions (finite $\mathrm{d} g_{0} / \mathrm{d} \mu$ and $g_{0}$ at $\mu= \pm 1$ ), we arrive at eqn (16) of the text. The corresponding displacement field is broken into the parallel and perpendicular components. The solution in the parallel direction is

$$
\begin{aligned}
d_{\|}\left(\mu ; \chi_{\mathrm{R}}\right)= & \frac{\mathrm{e}^{\mu \chi_{\mathrm{R}}}}{8 \pi\left(\sinh \chi_{\mathrm{R}}\right)^{2}}\left[\cosh \left(\chi_{\mathrm{R}}\right) \log \left(\frac{1-\mu}{1+\mu}\right)\right. \\
& -\sinh \left(\chi_{\mathrm{R}}\right) \log \left(1-\mu^{2}\right)+\mathrm{e}^{\chi_{\mathrm{R}}} \operatorname{Ei}\left(-\chi_{\mathrm{R}}(\mu+1)\right) \\
& \left.-\mathrm{e}^{-\chi_{\mathrm{R}}} \operatorname{Ei}\left(\chi_{\mathrm{R}}(1-\mu)\right)\right]+A_{\|} \mathrm{e}^{\mu \chi_{\mathrm{R}}},
\end{aligned}
$$
where $\operatorname{Ei}(t)$ is the exponential integral $\operatorname{Ei}(t) \equiv \int_{-\infty}^{t} \mathrm{e}^{-\zeta} / \zeta \mathrm{d} \zeta$, and
$A_{\|}$is the normalization constant:

$$
\begin{aligned}
A_{\|}= & -\frac{\chi_{\mathrm{R}}}{16 \pi\left(\sinh \chi_{\mathrm{R}}\right)^{3}} \int_{-1}^{1} \mathrm{e}^{\mu \chi_{\mathrm{R}}}\left[\cosh \left(\chi_{\mathrm{R}}\right) \log \left(\frac{1-\mu}{1+\mu}\right)\right. \\
& -\sinh \left(\chi_{\mathrm{R}}\right) \log \left(1-\mu^{2}\right)+\mathrm{e}^{\chi_{\mathrm{R}}} \operatorname{Ei}\left(-\chi_{\mathrm{R}}(\mu+1)\right) \\
& \left.-\mathrm{e}^{-\chi_{\mathrm{R}}} \operatorname{Ei}\left(\chi_{\mathrm{R}}(1-\mu)\right)\right] \mathrm{d} \mu .
\end{aligned}
$$

In the perpendicular direction, the solution is expanded as $d_{\perp}=\sum_{n=1}^{\infty} C_{n} P_{n}^{1}(\mu)$. The coefficients $C_{n}$ are found by solving a tridiagonal matrix problem:

$$
-\chi_{\mathrm{R}} \frac{(n+1)(n-1)}{2 n-1} C_{n-1}+n(n+1) C_{n}+\chi_{\mathrm{R}} \frac{n(n+2)}{2 n+3} C_{n+1}=b_{n},
$$

with $C_{0}=0$, and the forcing coefficients $b_{n}$ are given by

$$
b_{n}=-\frac{2 n+1}{2 n(n+1)} \int_{-1}^{1} g_{0}\left(\mu ; \chi_{\mathrm{R}}\right) \sqrt{1-\mu^{2}} P_{n}^{1} \mathrm{~d} \mu .
$$

From eqn (9), the swim diffusivity and stress are

$$
\begin{aligned}
\boldsymbol{\sigma}^{\text {swim }}= & -n \zeta\left\langle\boldsymbol{D}^{\text {swim }}\right\rangle=-n \zeta U_{0}^{2} \tau_{\mathrm{R}} \pi \int_{-1}^{1}\left[2 d _ { \| } \left(\operatorname{coth} \chi_{\mathrm{R}}\right.\right. \\
& \left.\left.-\chi_{\mathrm{R}}{ }^{-1}-\mu\right) \hat{\boldsymbol{H}} \hat{\boldsymbol{H}}+d_{\perp} \sqrt{1-\mu^{2}}(\boldsymbol{I}-\hat{\boldsymbol{H}} \hat{\boldsymbol{H}})\right] \mathrm{d} \mu,
\end{aligned}
$$

where only the diagonal terms contribute to the quadrature. In the perpendicular direction, the convenience of using associated Legendre polynomials is evident in

$$
\begin{aligned}
\sigma_{\perp}^{\text {swim }} & =-n \zeta U_{0}^{2} \tau_{\mathrm{R}} \pi \int_{-1}^{1} \sum_{n=1}^{\infty} C_{n} P_{n}^{1}(\mu) P_{1}^{1}(\mu) \mathrm{d} \mu \\
& =-\frac{4 \pi}{3} n \zeta U_{0}{ }^{2} \tau_{\mathrm{R}} C_{1} .
\end{aligned}
$$

\section{(D) Exact solution for arbitrary $\chi_{\mathrm{R}}$ : nonuniform speeds}

Resolving eqn (19) into the parallel and perpendicular components, the exact $\boldsymbol{d}$-field solution in the parallel direction is

$$
\begin{aligned}
d_{\|}= & \frac{\mathrm{e}^{\mu \chi_{\mathrm{R}}}}{8 \pi\left(\sinh \chi_{\mathrm{R}}\right)^{2}}\left\{( 1 - \frac { 2 \alpha H _ { 0 } } { \chi _ { \mathrm { R } } } ) \left[\cosh \left(\chi_{\mathrm{R}}\right) \log \left(\frac{1-\mu}{1+\mu}\right)\right.\right. \\
& -\sinh \left(\chi_{\mathrm{R}}\right) \log \left(1-\mu^{2}\right)+\mathrm{e}^{\chi_{\mathrm{R}}} \operatorname{Ei}\left(-\chi_{\mathrm{R}}(\mu+1)\right) \\
& \left.\left.-\mathrm{e}^{-\chi_{\mathrm{R}}} \operatorname{Ei}\left(\chi_{\mathrm{R}}(1-\mu)\right)\right]-2 \alpha H_{0} \mu \sinh \chi_{\mathrm{R}}\right\}+\tilde{A}_{\|} \mathrm{e}^{\mu \chi_{\mathrm{R}}},
\end{aligned}
$$

where $\tilde{A}_{\|}$is found from the normalization constraint to be

$$
\begin{aligned}
\tilde{A}_{\|}= & -\frac{\chi_{\mathrm{R}}}{16 \pi\left(\sinh \chi_{\mathrm{R}}\right)^{3}} \int_{-1}^{1} \mathrm{e}^{\mu \chi_{\mathrm{R}}}\left\{\left(1-\frac{2 \alpha H_{0}}{\chi_{\mathrm{R}}}\right)\right. \\
& \times\left[\cosh \left(\chi_{\mathrm{R}}\right) \log \left(\frac{1-\mu}{1+\mu}\right)-\sinh \left(\chi_{\mathrm{R}}\right) \log \left(1-\mu^{2}\right)\right. \\
& \left.+\mathrm{e}^{\chi_{\mathrm{R}}} \operatorname{Ei}\left(-\chi_{\mathrm{R}}(\mu+1)\right)-\mathrm{e}^{-\chi_{\mathrm{R}}} \operatorname{Ei}\left(\chi_{\mathrm{R}}(1-\mu)\right)\right] \\
& \left.-2 \alpha H_{0} \mu \sinh \chi_{\mathrm{R}}\right\} \mathrm{d} \mu .
\end{aligned}
$$

Substitution of this equation into eqn (9) gives the swim stress in the parallel direction.

In the perpendicular direction, the form of the solution is the same as before (eqn (C4)) except the forcing coefficients $b_{n}$ are given by

$$
b_{n}=-\frac{2 n+1}{2 n(n+1)} \int_{-1}^{1} g_{0}\left(\mu ; \chi_{\mathrm{R}}\right) \sqrt{1-\mu^{2}}\left(1+\alpha H_{0} \mu\right) P_{n}^{1} \mathrm{~d} \mu .
$$

The tridiagonal matrix problem is solved for the coefficients $C_{n-1}, C_{n}$, and $C_{n+1}$. The effective translational diffusivity in the perpendicular direction is given by

$$
\left\langle D_{\perp}\right\rangle=4 \pi U_{0}^{2} \tau_{\mathrm{R}}\left(\frac{1}{3} C_{1}+\frac{1}{5} \alpha H_{0} C_{2}\right),
$$

where we have used the orthogonality of the associated Legendre functions $P_{1}^{1}=-\sqrt{1-\mu^{2}}$ and $P_{2}^{1}=-3 \mu \sqrt{1-\mu^{2}}$ to evaluate the integral. 


\section{Acknowledgements}

SCT is supported by the Gates Millennium Scholars fellowship and the National Science Foundation Graduate Research Fellowship under Grant no. DGE-1144469.

\section{References}

1 S. Ramaswamy, Annu. Rev. Condens. Matter Phys., 2010, 1, 323-345.

2 S. C. Takatori, W. Yan and J. F. Brady, Phys. Rev. Lett., 2014, 113, 028103.

3 J. Adler, Science, 1966, 153, 708-716.

4 J. Armitage and K. Hellingwerf, Photosynth. Res., 2003, 76, 145-155.

5 R. Blakemore, Science, 1975, 190, 377-379.

6 D. Hader, R. Hemmersbach and M. Lebert, Gravity and the Behavior of Unicellular Organisms, Cambridge University Press, Cambridge, United Kingdom, 2005.

7 M. Doi, Soft Matter Physics, Oxford University Press, Oxford, United Kingdom, 2013.

8 D. S. Fudge, T. Winegard, R. H. Ewoldt, D. Beriault, L. Szewciw and G. H. McKinley, Integr. Comp. Biol., 2009, 49, 32-39.

9 S. K. Lai, Y.-Y. Wang, D. Wirtz and J. Hanes, Adv. Drug Delivery Rev., 2009, 61, 86-100.

10 D. Saintillan and M. J. Shelley, Phys. Fluids, 2008, 20, 123304123315.

11 T. Ishikawa, M. P. Simmonds and T. J. Pedley, J. Fluid Mech., 2006, 568, 119-160.

12 I. Theurkauff, C. Cottin-Bizonne, J. Palacci, C. Ybert and L. Bocquet, Phys. Rev. Lett., 2012, 108, 268303.

13 J. Palacci, S. Sacanna, A. P. Steinberg, D. J. Pine and P. M. Chaikin, Science, 2013, 339, 936-940.
14 H. Brenner, J. Colloid Interface Sci., 1979, 71, 189-208.

15 Y. Almog and I. Frankel, J. Colloid Interface Sci., 1993, 157, 60-71.

16 M. A. Bees, N. A. Hill and T. J. Pedley, J. Math. Biol., 1998, 36, 269-298.

17 A. Manela and I. Frankel, J. Fluid Mech., 2003, 490, 99-127.

18 T. J. Pedley and J. O. Kessler, J. Fluid Mech., 1990, 212, 155182.

19 R. N. Bearon, A. L. Hazel and G. J. Thorn, J. Fluid Mech., 2011, 680, 602-635.

20 R. N. Bearon, M. A. Bees and O. A. Croze, Phys. Fluids, 2012, 24, 121902-121920.

21 M. Demir and H. Salman, Biophys. J., 2012, 103, 1683-1690.

22 R. R. Vuppula, M. S. Tirumkudulu and K. V. Venkatesh, Phys. Biol., 2010, 7, 026007.

23 R. N. Zia and J. F. Brady, J. Fluid Mech., 2010, 658, 188210.

24 U. M. Córdova-Figueroa and J. F. Brady, Phys. Rev. Lett., 2008, 100, 158303.

$25 \mathrm{H}$. Brenner and D. Edwards, Macrotransport Processes, Butterworth-Heinemann Limited, 1993.

26 H. Brenner and D. W. Condiff, J. Colloid Interface Sci., 1974, 47, 199-264.

27 H. Brenner, Chem. Eng. Sci., 1964, 19, 631-651.

28 D. A. Beard and T. Schlick, Biophys. J., 2003, 85, 29732976.

29 J. Toner, Y. Tu and S. Ramaswamy, Ann. Phys., 2005, 318, 170-244.

30 A. Snezhko and I. S. Aranson, Nat. Mater., 2011, 10, 698-703.

31 M. V. Sapozhnikov, Y. V. Tolmachev, I. S. Aranson and W. K. Kwok, Phys. Rev. Lett., 2003, 90, 114301.

32 M. E. Leunissen, H. R. Vutukuri and A. van Blaaderen, Adv. Mater., 2009, 21, 3116-3120. 